
\title{
R Research Sourare \\ Single cell profiling of transcriptomic changes during in vitro maturation of human oocytes
}

\author{
Hiroki Takeuchi \\ Mie University \\ Mari Yamamoto \\ Kindai University \\ Megumi Fukui \\ Mie University \\ Tadashi Maezawa \\ Mie University \\ Mikiko Nishioka \\ Mie University \\ Eiji Kondo \\ Mie University \\ Tomoaki Ikeda \\ Mie University \\ Kazuya Matsumoto \\ Kindai University \\ Kei Miyamoto ( $\boldsymbol{\sigma}$ kmiyamo@waka.kindai.ac.jp ) \\ Kindai University
}

\section{Research Article}

Keywords: Human oocyte, Single cell RNA-sequencing, Oocyte maturation, Transcriptome

Posted Date: March 17th, 2021

DOI: https://doi.org/10.21203/rs.3.rs-292144/v1

License: (c) (1) This work is licensed under a Creative Commons Attribution 4.0 International License.

Read Full License 
2 Single cell profiling of transcriptomic changes during in vitro maturation 3 of human oocytes

5 Hiroki Takeuchi ${ }^{1, a}$, Mari Yamamoto $^{2, a}$, Megumi Fukui ${ }^{1}$, Tadashi Maezawa ${ }^{1}$, Mikiko Nishioka ${ }^{1}$, Eiji

6 Kondo $^{1}$, Tomoaki Ikeda ${ }^{1}$, Kazuya Matsumoto ${ }^{2}$ and Kei Miyamoto ${ }^{2 *}$

7

$8{ }^{1}$ Department of Obstetrics and Gynecology, Graduate School of Medicine, Mie University, Mie 514-8507,

9 Japan.

$10 \quad{ }^{2}$ Graduate School of Biology-Oriented Science and Technology, Kindai University, Wakayama 649-6493, 11 Japan.

12

13 aThese authors contributed equally to this work

14

15 *Corresponding author: Kei Miyamoto

16 E-mail:kmiyamo@waka.kindai.ac.jp

17 Telephone: +81-736-77-0345(EXT.4405)

18 FAX: +81-736-77-4754

19 


\section{Abstract}

21 In vitro maturation of human oocytes is widely used for infertility treatment. However, the success rate

22 of maturation varies depending on patients and molecular mechanisms underlying successful maturation

23 remain unclear. Especially, gene expression profiles of oocytes at each maturation stage need to be

24 revealed to understand the differential developmental abilities of oocytes. Here, we show transcriptomes

25 of human oocytes during in vitro maturation by single cell RNA-seq analyses. Hundreds of transcripts

26 dynamically altered their expression, and we identify molecular pathways and upstream regulators that

27 may govern oocyte maturation. Furthermore, oocytes that are delayed in their maturation show distinct

28 transcriptomes. Finally, we reveal genes whose transcripts are enriched in each maturation stage and that

29 can be used for selecting an oocyte with a high developmental potential. Taken together, our work

30 uncovers transcriptomic changes during human oocyte maturation and provides a molecular insight into

31 the differential developmental potential of each oocyte.

32

\section{$33 \quad$ Keywords}

34 Human oocyte; Single cell RNA-sequencing, Oocyte maturation; Transcriptome 


\section{Introduction}

37 The first successful pregnancy of an in vitro matured human oocyte after in vitro fertilization was reported in $1991^{1}$. Although the clinical application of in vitro maturation (IVM) has increased since this report $^{2}$, IVM technology is not widely used in infertility treatment due to its poor outcome. Importantly, it is generally regarded that the quality of embryos generated from IVM oocytes is worse than that of embryos from in vivo matured oocytes in multiple aspects, such as developmental competence and subsequent pregnancy rates ${ }^{3,4}$. Therefore, oocytes other than the metaphase II (MII) stage at the time of oocyte retrieval are discarded in many fertility clinics. However, IVM of immature oocytes offers a unique opportunity. For women diagnosed with polycystic ovary syndrome, IVM of non-MII oocytes can prevent the risk of ovarian hyperstimulation syndrome. In addition, ovarian tissue is cryopreserved prior to cancer treatment for ensuring fertility of patients who might lose gonadal functions due to the treatment. The introduction of this fertility preservation techniques has provided the possibility of obtaining immature oocytes from very small antral follicles and cryopreserving them after in vitro maturation ${ }^{5}$. There are reports of pregnancy from embryos obtained from IVM of these immature oocytes $^{6,7,8}$. Thus, IVM of immature oocytes plays a key role in warranting fertility for some patients. Despite the fact that IVM of human oocytes is an invaluable choice for human reproductive technologies, the pregnancy rate of IVM-derived oocytes is still low ${ }^{3,9}$. Moreover, the developmental 53 ability of IVM oocytes is variable ${ }^{3}$. It is extremely important to learn about the nature of this variability 54 of oocyte quality. The quality of oocytes is profoundly affected by gene expression during oocyte 55 growth ${ }^{10,11}$. As a result, the gene expression pattern of MII oocytes with a high developmental capacity 56 seems different from that with a low developmental ability ${ }^{12,13}$. However, it remains unclear when the gene expression pattern of human MII oocytes is established during maturation and how variable transcriptomes are found among different individuals. 
microarrays ${ }^{14,15,16}$. One report has revealed that in vitro-matured MII oocytes show the over-abundance of detected transcripts, as compared with in vivo-derived MII oocytes ${ }^{15}$, suggesting a different transcriptome pattern between in vitro- and in vivo-matured oocytes. It has also suggested that GV and MI oocytes show similar gene expression profiles ${ }^{15}$. Recently, single cell RNA-seq has been applied to reveal transcriptomes of human oocytes ${ }^{17,18,19}$. Zhang et al. reported transcriptomic profiles of human oocytes during folliculogenesis ${ }^{18}$. Differential gene expression related to energy metabolism has been identified between in vitro- and in vivo-matured oocytes ${ }^{19}$. However, transcriptomic changes during in vitro oocyte maturation from the germinal vesicle $(\mathrm{GV})$ stage till the MII stage have not been well elucidated even though these oocytes are often collected after oocyte retrieval for infertility treatment. Furthermore, transcriptomic variations among different individuals need to be determined.

In this study, transcriptomes for oocytes at different maturation stages were examined using single cell RNA-seq and differentially expressed genes were identified. Surplus oocytes, which are discarded as medical waste, were subjected to this study. Immature oocytes at the time of oocyte retrieval were used for in vitro maturation, and oocytes at the GV, metaphase I (MI), and MII stages were subjected to RNA-seq. Moreover, oocytes that reached each maturation stage with a significant delay were also collected for the comparison. By comparing the transcriptomes of GV, MI, and MII oocytes, we found genes and pathways whose expression was dynamically altered during maturation. Furthermore, we compared the oocyte transcriptomes obtained from different patients and reveal the gene set that tends to be differentially expressed among individuals. Our study deepens the molecular understanding of human oocyte maturation and provides an important platform to search for maternal genes that reflect the oocyte quality. 
RNA-seq analyses of human oocytes during oocyte maturation.

85 To reveal transcriptomes of human oocytes during in vitro maturation, single cell RNA-seq analyses

86 were performed using oocytes at different maturation stages. In total, 19 oocyte samples, collected from

8711 patients, were subjected to RNA-seq analyses. Oocytes were classified into three different maturation stages; the immature GV stage, maturing oocytes from the GV to MII stage, and the matured MII stage

(Fig. 1a). Maturing oocytes from the GV to MII stage were described as the metaphase I (MI), and many of them showed condensed chromosomes after DNA staining (Fig. 1a, b). As shown in Fig. 1a, human oocytes were first collected for infertility treatment and oocytes at the MII stage or that reached the MII stage until 0-7 hours after the oocyte retrieval were used for in vitro fertilization. Oocytes that did not reach the MII stage by then are routinely discarded as medical wastes in our hospital (Mie university)

94 and these surplus oocytes were analyzed. On the same day of oocyte retrieval, oocytes at the GV, MI and

95 MII stages were morphologically judged at 7.5-9 hours after oocyte retrieval (GV, MI, and MII, respectively) and sampling was carried out (Fig. 1a). Some remaining oocytes were further cultured for 15-16.5 hours, and oocytes that reached each developmental stage were collected. These oocytes were called as delayed GV, MI and MII oocytes (dGV, dMI, and dMII, respectively). Successful collection of oocytes at different maturation stages were confirmed with DNA staining (Fig. 1b). The collected oocytes were subjected to single cell RNA-seq by taking advantage of the SMART-seq procedure ${ }^{20}$.

101 After sequencing, 13,799,742 $\pm 4,127,048$ reads were obtained and $85.6 \pm 2.0 \%$ of reads were uniquely

102 mapped to the human hg19 genome (Supplementary Table 1). When the reads and mapping rates were 103 calculated in each oocyte maturation stage, similar statistics were obtained (Supplementary Table 1).

104 We then compared transcriptomes during oocyte maturation. Generally, oocytes derived from 105 the same maturation stage resemble each other in their transcriptomes (Supplementary Fig. 1). Principal 106 component analysis (PCA) captured transcriptomic transitions during human oocyte maturation (Fig. 1c). 107 Oocytes at different maturation stages were also separated by a hierarchical clustering analysis (Fig. 1d). 
108 Of note, a small number of oocytes were not well classified into the corresponding oocyte stages; GV3

109 rather exhibited a higher correlation with MI oocytes (Fig. 1c, d and Supplementary Fig. 1) and MII1

110 showed a closer transcriptome to that of the GV or MI state (Fig. 1c, d and Supplementary Fig. 1). These

111 results suggest that an oocyte classified as the MII stage according to the morphological criteria can

112 contain an improperly matured oocyte at the transcriptome level.

113 We next sought to identify differentially expressed genes (DEGs) among different maturation 114 stages. When a stringent criterion is used (padj $<0.05$, 4-fold difference in FRKM values), 1, 8, and 8 115 DEGs were identified by comparing GV vs MI, MI vs MII, and GV vs MII stage oocytes, respectively. 116 This is probably due to the variable transcriptome of some individual oocytes such as MII1 and GV3 117 (Fig. 1c, 1d and Supplementary Fig. 1). Then, we used another condition to find DEGs between different 118 oocyte maturation stages ( $P<0.05$, 4-fold difference in FRKM values), and 241, 428, and 523 DEGs 119 were identified by comparing GV vs MI, MI vs MII, and GV vs MII stage oocytes, respectively. DEGs 120 were further grouped into upregulated and downregulated genes in each comparison (Fig. 1e, f); 77 (GV 121 vs MI), 317 (MI vs MII), and 360 (GV vs MII) upregulated genes were found, while 164 (GV vs MI), 122111 (MI vs MII), and 163 (GV vs MII) genes were downregulated (Supplementary Table 2). Genes that 123 were previously shown to be involved in oocyte meiosis and subsequent embryonic development such as $124 A S F 1 B$ and DPPA3 were indeed upregulated in MII oocytes (Table S2) ${ }^{21,22}$. GO analyses were performed 125 using these gene sets (Supplementary Fig. 2a-c). Interestingly, many specific terms were found by 126 comparing to MII transcriptomes. Some GO terms such as MAP kinase activity were also found in the 127 previous study in which human GV and MII transcriptome were compared ${ }^{23}$. Expression of genes related 128 to lipoprotein metabolism was significantly altered between maturing MI and MII stages 129 (Supplementary Fig. 2b). Abnormal lipoprotein metabolism is relevant to female infertility ${ }^{24}$, suggesting 130 that expression of important genes for subsequent development is dynamically changed at this 131 maturation stage. Moreover, when DEGs found between GV and MII were analyzed, myosin-related 
132 terms were found, in good agreement with the previous report that Myosin IIA, IIB and microfilaments

133 are dynamically reorganized during oocyte maturation ${ }^{25}$. Our DEG list contains myosins (MYH3, 134 MYH4, MYH8) and tubulins (TUBA1A, TUBA1B, TUBA1C, TUBA3E, TUBA3D) as upregulated 135 genes in MII oocytes (Fig. 1g). Translational regulation of these genes might be key for in vitro oocyte 136 maturation.

137 To further characterize transcriptomic changes during human oocyte maturation, DEGs 138 identified by comparing each maturation stage were subjected to Ingenuity Pathway Analysis (IPA). IPA 139 of DEGs revealed the significantly changed canonical pathways and predicted upstream regulators. 140 Many canonical pathways were found as significantly altered by comparing GV vs MI or GV vs MII 141 stage oocytes (Fig. 2a). As upstream regulators, AKT (also called protein kinase B) was identified by 142 comparing GV and MI transcriptomes (Fig. 2b). The previous report also showed a dynamic change of 143 AKT activity for the meiotic progression of GV oocytes to the MI stage ${ }^{26}$. When GV and MII 144 transcriptomes were compared, NOBOX, TEAD3, PSEN1 were identified as top upstream regulators 145 (Supplementary Table 3), suggesting that genes regulated by these transcription factors might 146 dynamically change their expression during in vitro maturation. Moreover, the lipid metabolic pathway 147 was again identified also by IPA as significantly affected during maturation (Supplementary Fig. 3). 148 Interestingly, hypoxia-inducible factor 1a (HIF1A) target genes were largely inhibited presumably due to 149 the effect of maternal HIF1A (Fig. 2c). HIF1A is involved in the hypoxia-induced dormant state of 150 oocytes $^{27}$ and is upregulated from the primary oocytes stage ${ }^{18}$. Injection of an inhibitor that suppresses 151 HIF1A activity into mice reduces the quality of oocytes by impairing the meiotic apparatus, suggesting a 152 defect in spindle assembly and actin dynamics ${ }^{28}$. In good agreement with this paper ${ }^{28}$, we have also 153 identified genes related to actin dynamics such as VASP and ACTA2 as downstream targets for HIF1A 154 (Fig. 2c). HIF is a key player for metabolic reprogramming in concert with Sirtuin ${ }^{29}$. Sirt1-Nrf2-Cyclin 155 B1/CDK1 signaling pathway is important for oocyte meiosis by regulating spindle/chromosome 
156 organization ${ }^{30}$. Importantly, canonical pathway analyses showed that Sirtuin signaling genes were

157 enriched in DEGs between GV and MII (Fig. 2a; GV vs MII, green arrow). Taken together, HIF1A and

158 Sirtuin signaling pathway might be an important player for in vitro oocyte maturation in human.

159

160

\section{Differentially expressed genes between normal and delayed oocytes}

161 We next examined transcriptomic profiles of oocytes that were significantly delayed to reach MI and MII stages (Fig. 1a; dMI and dMII, respectively) or GV oocytes that remained immature (Fig. 1a; dGV). These types of oocytes are not used for human infertility treatment, but their developmental ability and similarity to the normally obtained oocytes have not been elucidated well. PCA analyses showed that transcriptomes of $\mathrm{dGV}$ were similar to those of GV oocytes (Fig. 3a, $+\mathrm{dGV}$ ), suggesting that transcripts are relatively stable during the overnight incubation of GV oocytes. In contrast, dMI samples were well separated from MI (Fig. 3a, +dMI). Regarding transcriptomes of dMII, two of them were close to MII, but one of dMII samples (dMII3) showed a distinct transcriptomic pattern (Fig. 3a, +dMII), suggesting that delayed MII oocytes include those close to MII oocytes at least in a transcriptomic level. A hierarchical clustering analysis also supported these notions (Supplementary Fig. 4a).

We then identified DEGs specifically up- or down-regulated in delayed oocytes. When a

172 stringent condition is used (padj $<0.05$, 4-fold difference in FRKM values), 1, 7, and 28 DEGs were 173 identified by comparing GV vs dGV, MI vs dMI, and MII vs dMII stage oocytes, respectively. Then, we 174 used another condition to find DEGs between different oocyte maturation stages $(P<0.05,4$-fold 175 difference in FRKM values), and 180, 568, and 414 DEGs were identified by comparing GV vs dGV, MI 176 vs dMI, and MII vs dMII stage oocytes, respectively. DEGs were further grouped into upregulated and 177 downregulated genes in each comparison (Fig. 3b, c, and Supplementary Table 4); 114 (GV vs dGV), 178 430 (MI vs dMI), and 119 (MII vs dMII) upregulated genes were found, while 66 (GV vs dGV), 138 (MI vs dMI), and 295 (MII vs dMII) genes were downregulated. GO analyses were performed using these 
180 gene sets (Supplementary Fig. 4b-d). Many specific terms were identified in downregulated genes in

181 dMI samples, and especially genes involved in ubiquitin proteasome pathways were misregulated

182 (Supplementary Fig. 4c). Moreover, the comparison between MII and dMII shows that apoptosis-related 183 genes were misregulated in delayed oocytes (Supplementary Fig. 4d). IPA of DEGs revealed canonical 184 pathways and predicted upstream regulators, which were significantly affected by delayed maturation. In 185 canonical pathway analysis, altered pathways between MII and dMII (Fig. 4a) were significantly 186 overlapped with the molecular pathways identified by comparing GV and MII (Fig. 2a). This result 187 suggests that genes and related pathways, which are activated during in vitro maturation, tend to be 188 misregulated in delayed MII oocytes. The term, G1/S Checkpoint Regulation, was only found when MII 189 and dMII sample were compared. CDKN1A gene is a part of the G1/S Checkpoint Regulation, and has 190 been shown to play a key role for oocyte meiosis ${ }^{31}$. CDKN1A was downregulated in delayed MII oocytes 191 (Supplementary Table 4), and thus might be used as a marker for selecting normally maturating oocytes. 192 Furthermore, genes related to Sirtuin Signaling Pathway were identified by comparing MII vs dMII 193 stage oocytes (Fig. 4a, green arrow); Of note, $C Y C 1, S D H D$, and tubulin genes (TUBA1A, TUBA1B, $194 T U B A 3 C / T U B A 3 D, T U B A 3 E, T U B A 4 A)$, all involved in Sirtuin Signaling Pathway, were downregulated 195 in delayed MII oocytes (Fig. 4b and Supplementary Table 4). As the upstream regulators, SMARCA4 196 was detected in the comparison between MII and dMII (Supplementary Table 5) and target genes of 197 SMARCA4 were downregulated (Fig. 4b). In good line with our observation, SMARCA4 was shown to 198 be accumulated in aged preovulatory oocytes ${ }^{32}$. Oocytes of delayed maturation might accumulate 199 SMARCA4, which in turn results in misregulated gene expression.

A specific set of genes are differentially expressed among different patients during oocyte maturation

203 We next sought to characterize genes that are variably expressed among MII oocytes of different patients. 
204 By comparing MII1 vs MII2, MII2 vs MII3, and MII3 vs MII1 oocytes, 475, 134, and 469 DEGs were

205 identified, respectively ( $P<0.05,4$-fold difference in FRKM values). DEGs were further grouped into

206 upregulated and downregulated genes in each comparison (Fig. 5a, b); 357 (MII1 vs MII2), 44 (MII2 vs

207 MII3), and 123 (MII3 vs MII1) upregulated genes were found, while 118 (MII1 vs MII2), 90 (MII2 vs

208 MII3), and 346 (MII3 vs MII1) genes were downregulated (Supplementary Table 6). PCA showed that

209 the MII1 transcriptome was distinct from other two MII samples (Fig. 1c). The heatmap also supports

210 the notion that the MII1 transcriptome was different from MII2 and MII3 (Fig. 5a, b), and rather similar

211 to delayed oocyte samples, dMII1 and dMII2. When all DEGs were compared on heatmap, the different

212 transcriptomic pattern of MII1 was obvious (Supplementary Fig. 5a). IPA revealed that genes related to

213 Sirtuin Signaling Pathway and Nucleotide Excision Repair (NER) Pathway were commonly

214 misregulated in MII1 oocytes (Fig. 5c: green arrows; Supplementary Table 7). Furthermore, KLF3 and

215 TET2 were identified as candidate upstream regulators for explaining this difference in transcriptomes

216 (Supplementary Fig. 5b, c) KLF3 controls the accumulation of fats and lipids ${ }^{33}$. Lipids are accumulated

217 in the oocyte cytoplasm during maturation ${ }^{34}$ and the synthesis and maintenance of lipid droplets are key

218 for the quality of the oocyte and subsequent embryonic development ${ }^{35}$. Tet 2 knockout mice shows

219 defects in polar body extrusion, mitotic spindle structure, and chromosomal alignment during meiosis,

220 all of which become apparent with aging, eventually resulting in the reduced oocyte quality and 221 embryonic development ${ }^{36}$. Taken together, specific gene pathways are disturbed in some of the in vitro 222 matured MII oocytes.

224 Potential marker genes for each oocyte maturation stage and for selecting a high quality oocyte

225 We next sought to find marker genes that were specifically or abundantly expressed in each maturation 226 stage of oocytes, focusing on GV and MII stages. We first performed unsupervised clustering of genes 227 based on their expression levels and 20 clusters were generated (Fig. 6a). For identifying GV-enriched 
228 transcripts, we focused on the cluster 5, which showed strong expression in GV samples, especially in 229 GV1 and GV2 (Fig. 6a). These genes were compared to our upregulated DEG list in GV samples (GV vs 230 MI and GV vs MII), and 40 genes were identified (Fig. 6b and Supplementary Table 8). EIF4EBP1 is 231 included in this list, which is known to be associated with maternal mRNA in mouse oocytes ${ }^{37}$. We next searched for transcripts uniquely expressed in matured MII oocytes. Clusters 9,12 , and 16 showed strong expression in MII oocytes, especially in MII2 and MII3 (Fig. 6a). When genes belonging to these clusters were compared to our upregulated DEG list in MII samples (GV vs MII and MI vs MII), 58 genes were identified (Fig. 6c, 32+15+11 genes). Alternatively, our MII DEGs were compared to human MII oocyte-specific genes previously identified by Zhao et al. ${ }^{23}$, and 8 overlapping genes were detected (Supplementary Fig. 6a and Supplementary Table 9). Taken together, these genes (Supplementary Table 9) can serve as MII-enriched markers during oocyte maturation. We then examined expression of these marker genes in delayed MII samples, which are supposed to show a lower developmental ability than normally matured MII oocytes. Interestingly, all of these marker genes were 241 downregulated in delayed MII oocytes (Fig. 6d), suggesting that these are good candidates as a marker 242 to select MII oocytes with a high quality. Especially, $A S F 1 B$ and BMP15 were highly expressed only in 243 MII2 and MII3 samples (Fig. 6d ${ }^{38,39}$, suggesting that these genes are good candidates for marker genes 244 to select MII oocytes with a high quality. Finally, we performed unsupervised clustering of MII and dMII 245 samples (Supplementary Fig. 6b), and clusters 6 and 12 were identified as highly abundant in MII2 and 246 MII3 samples (Supplementary Table 10). APOB and TNFRSF10C were identified as MII-enriched 247 markers, and therefore might serve as a biomarker for selecting MII oocytes with a high quality. $A P O B$ 248 knockout mice have been reported to be embryonic lethality in homozygous ${ }^{40}$. In addition, it has been 249 reported that a positive correlation is observed between the APOB concentration in follicular fluid and 250 the embryo quality such as fertilization rates and embryonic developmental potentials ${ }^{41}$. Furthermore, 251 TNFRSF10C (TRAILR3) protein is decreased from a medium containing human blastocysts, suggesting 
252 TNFRSF10C is utilized for embryonic development ${ }^{42}$. Taken together, we have not only identified

253 specific marker transcripts for each maturation state of oocytes, but also shown potential biomarkers to 254 select a high quality human oocyte for subsequent development. 


\section{Methods}

Human oocyte. Informed consent for this prospective study was obtained from all participants. The oocyte samples used in this study were collected from 13 patients in 2019, and these oocyte samples are normally discarded as clinical waste after the completion of in vitro fertilization in clinical treatment. After aspirating follicles with a fine needle under the ovarian echo, the cumulus oocyte complexes (COCs) were picked up under a stereomicroscope. COCs from infertile patients were scheduled for intracytoplasmic sperm injection, and surrounding cumulus cells were removed in multipurpose handling medium (MHM; Scientific's, USA, 90163) containing $20 \mathrm{IU} / \mathrm{ml}$ Cumulus Remover (Recombinant hyaluronidase; Kitazato, Japan, 94114). After denudation, oocyte morphology was examined and an oocyte at the MII stage was used for infertility treatment. Oocytes other than the MII stage were subjected to in vitro maturation. The collected and denuded oocytes were incubated in the Sydney IVF fertilization medium (Cook medical, USA, K-sifm-100) at $37^{\circ} \mathrm{C}$ under $5 \% \mathrm{CO}_{2}$ and $5 \% \mathrm{O}_{2}$ in air for in vitro maturation, and oocytes that had matured to the MII stage by 16:00 on the same day of oocyte retrieval were used for the treatment of in vitro fertilization. The remaining oocytes that had not reached the MII stage by 16:00 were normally discarded, but further cultured for this study. A few hours after the incubation, some oocytes were matured to MII oocytes, and such matured oocytes were subjected to sampling for RNA-seq analyses. At that time, GV and MI oocytes were also sampled. For MII oocytes, zona pellucide piercing was performed on the denuded oocytes with LYKOS Clinical IVF laser system and Clinical Laser Software Legacy 5.12 (Hamilton Thorne, USA, 40025) and the first polar body was biopsied with micropipette (CooperSurgical, USA, MPBFP30). Zona was lysed with acidic-tyrode (Merck, Germany, T1788) for 20-30 seconds, and washed in Phosphate-buffered saline (PBS; Nacalai Tesque, Japan, 07269-84) containing 0.1\% w/v bovine serum albumin (BSA; Merck, Germany, NM-126609-5GMCN). The zona- and polar body-free oocytes suspended in $1 \mu \mathrm{lof} 0.1 \% \mathrm{w} / \mathrm{v}$ BSA/PBS were moved into $9.5 \mu 1$ of $1 \times$ Reaction buffer from SMART-seq v4 Ultra Low Input RNA Kit 
280 (Takara Bio, Japan, 24888N), in which 10× lysis buffer (Takara Bio, Japan, 635013) and 2 unit 281 ribonuclease inhibitor (Thermo Fisher Scientific, USA, 10777-019) were mixed. GV and MI oocytes 282 were similarly sampled for RNA-seq analyses, except that polar body removal was not carried out. Some 283 oocytes that remained immature at the time of the above mentioned sampling for RNA-seq analyses 284 were left in the maturation medium overnight, and we examined the maturation state on the next day of 285 the oocyte retrieval (9:00 am). Such oocytes were sampled as those which were delayed in their 286 maturation by following the same sampling method as described above. The classification criteria for GV, 287 MI and MII oocytes were explained in the corresponding result section. This study was performed within 288 the guidelines established by the Clinical Research Ethics Review Committee of Mie University 289 Hospital (H2018-066) and was registered in the University Hospital Medical Information Network 290 Clinical Trials Registry in Japan (UMIN000034811). In addition, this study was registered for the 291 research dealing with human sperm, ova, and fertilized ova in the Japan Society of Obstetrics and 292 Gynecology.

294 Nuclear staining. Nuclear staining was performed using the surplus oocytes at each stage as follows. 295 Oocytes were incubated in the fixation medium, 4\% w/v paraformaldehyde (Nacalai tesque, Japan, 296 26126-25), for $30 \mathrm{~min}$ at room temperature, followed by three washes with $0.2 \% \mathrm{w} / \mathrm{v}$ BSA/PBS. After 297 permeabilization with $0.2 \% \mathrm{v} / \mathrm{v}$ Triton X-100/PBS for $30 \mathrm{~min}$ at room temperature, they were washed 298 with $0.2 \% \mathrm{w} / \mathrm{v}$ BSA/PBS. Then, nuclei were stained with $1 \mu \mathrm{g} / \mu \mathrm{L}$ DAPI (Nacalai tesque, Japan, $29911034-56$ ) in $0.2 \% \mathrm{w} / \mathrm{v}$ BSA/PBS solution for $10 \mathrm{~min}$, followed by washes with $0.2 \% \mathrm{w} / \mathrm{v}$ BSA/PBS. 300 The fluorescence signals were observed using a confocal microscope (Carl Zeiss, Germany, LSM800), 301 equipped with a laser module (405/488/561/640 nm) and GaAsP detector. Z-slice thickness was 302 determined by using the optimal interval function in the ZEN software (Carl Zeiss). 
304 Library preparation for RNA-seq. A single oocyte at each maturation stage was subjected to cDNA 305 synthesis and amplification using SMART-Seq ${ }^{\circledR}$ v4 Ultra ${ }^{\circledR}$ Low Input RNA Kit (Takara Bio, Japan, 306 Z4889N) according to the vendor's instruction with some modifications. Briefly, collected surplus 307 oocytes were subjected to micromanipulation to remove their sibling polar bodies, and then zona 308 pellucida was enzymatically removed as described above. After removing the polar body and the zona 309 pellucida, oocytes were washed with $0.1 \% \mathrm{w} / \mathrm{v}$ BSA/PBS, and then transferred to a $0.2 \mathrm{ml}$ tube 310 containing $9.5 \mu \mathrm{l}$ of reaction buffer to make the final volume of $10.5 \mu \mathrm{l}$. After cell lysis in the buffer, $1 \mu \mathrm{l}$ 311 of the solution was removed and, instead, $1 \mu \mathrm{l}$ of the diluted ERCC RNA Spike-In Mix was added 312 (diluted for $350 \mathrm{pg}$ of total RNA according to the vender's instruction; Thermo Fisher Scientific, 313 4456740). The lysed RNA solution with the spike-in RNA was subjected to reverse transcription. The 314 produced cDNA was amplified by PCR with 16 cycles. The amplified cDNA was purified using AMPure 315 XP beads (Beckman Coulter, USA, A63882). The purified cDNA was measured on the Bioanalyzer 316 (Agilent, USA) using High Sensitivity DNA Kit (Agilent, 5067-4626) and the normalized volume of 317 DNA was subjected to library preparation using Nextera XT DNA Library Preparation Kit (Illumina, 318 USA, FC-131-1024) by following the vendor's instruction. The obtained library was quality-checked by 319 Bioanalyzer and quantified using Qubit (Thermo Fisher Scientific). The experiments have been 320 approved by the Clinical Research Ethics Review Committee of Kindai University at the Department of 321 Biology-Oriented Science and Technology (H30-1-006) and followed the university's guidelines to store 322 the sequencing data.

324 Sequencing data filtering. Paired end sequencing (50 bp $+25 \mathrm{bp})$ was carried out using the Illumina 325 NextSeq system. Fastq files from Illumina sequencing were filtered for low quality reads by sliding 326 window trimming (window size $10,<\mathrm{QV} 20$ ), and low quality bases were trimmed from the ends of the 327 reads $(<\mathrm{QV} 20)$ using Trimmomatic ${ }^{43}$. Reads of less than 20 bases and unpaired reads were removed. 
328 Furthermore, adaptor, polyA, polyT and polyG sequences were removed using trim_galore and 329 cutadapt $t^{44}$. The sequencing reads were then mapped to the human genome (hg19) using STAR ${ }^{45}$. Reads 330 on annotated genes were counted using featureCounts ${ }^{46}$.

RNA-seq analysis. RNA-seq reads were visualized using Integrative Genome Viewer ${ }^{47}$. FPKM values were calculated from mapped reads by normalizing to total counts and transcript. Differentially expressed genes (DEGs, $P<0.05$ ) were then identified using DESeq2 ${ }^{48}$. An unsupervised hierarchical clustering of the read count values was performed using hclust in TCC (unweighted pair group method with arithmetic mean: UPGMA). Gene ontology terms showing over-representation of genes that were up or down-regulated were detected using DAVID tools $(P<0.05)^{49,50}$. Furthermore, the gene list of DEGs was compared to the categorized gene lists found in a paper ${ }^{23}$. Each gene list was further subjected to an Ingenuity Pathway Analysis (IPA; Qiagen, Redwood City, USA, https://www.qiagenbioinformatics.com/products/ingenuity-pathway-analysis). Using IPA, enriched canonical pathways, upstream transcriptional regulators, gene regulatory networks, and diseases and biological functions in the identified DEGs were investigated. Expression levels of DEGs were

343 displayed in heatmaps with z-scores obtained from FPKM values of genes in the DEG lists by using 344 heatmapper ${ }^{51}$. The DEG lists consist of genes that satisfy the condition $P$ value $<0.05$ and $\log 2 \mathrm{FC}>2$ or $345 \quad \log 2 \mathrm{FC}<-2$.

347 Statistical and Reproducibility. Significant GO terms were identified using the modified Fisher Exact 348 P-values according to the DAVID functional annotation tool. In IPA, $P$ values were obtained according 349 to the statistical methods applied to each analysis explained in the vendor's instruction. A value of $P$ $350<0.05$ was considered significant. Number of replicates is indicated in the figure legends. 
352 Reporting summary. Further information on research design is available in the Nature Research 353 Reporting Summary linked to this article.

354

355

Data availability

356 The dataset generated during this study are available in the Supplementary Tables and in the Gene 357 Expression Omnibus (GEO) public repository under accession GSE166533. All the other data are 358 available from the corresponding author upon reasonable request.

359

360

361

362

363

364

365 


\section{References}

1. Cha, K. Y. et al. Pregnancy after in vitro fertilization of human follicular oocytes collected from nonstimulated cycles, their culture in vitro and their transfer in a donor oocyte program. Fertil Steril 55, 109-113 (1991).

2. Basatemur, E. \& Sutcliffe, A. Health of IVM children. J Assist Reprod Genet 28, 489-493 (2011).

3. Nogueira, D., Sadeu, J. C. \& Montagut, J. In vitro oocyte maturation: current status. Semin Reprod Med 30, 199-213 (2012).

4. Walls, M. L. et al. In vitro maturation as an alternative to standard in vitro fertilization for patients diagnosed with polycystic ovaries: a comparative analysis of fresh, frozen and cumulative cycle outcomes. Hum Reprod 30, 88-96 (2015).

5. Fasano, G., Moffa, F., Dechène, J., Englert, Y. \& Demeestere, I. Vitrification of in vitro matured oocytes collected from antral follicles at the time of ovarian tissue cryopreservation. Reprod Biol Endocrinol 9, 150 (2011).

6. Prasath, E. B. et al. First pregnancy and live birth resulting from cryopreserved embryos obtained from in vitro matured oocytes after oophorectomy in an ovarian cancer patient. Hum Reprod 29, 276-278 (2014).

7. Segers, I. et al. In vitro maturation (IVM) of oocytes recovered from ovariectomy specimens in the laboratory: a promising 'ex vivo' method of oocyte cryopreservation resulting in the first report of an ongoing pregnancy in Europe. J Assist Reprod Genet 32, 1221-1231 (2015).

8. Uzelac, P. S., Delaney, A. A., Christensen, G. L., Bohler, H. C. L. \& Nakajima, S. T. Live birth following in vitro maturation of oocytes retrieved from extracorporeal ovarian tissue aspiration and embryo cryopreservation for 5 years. Fertil Steril 104, 1258-1260 (2015).

9. Chen, S. U. et al. Schedule to inject in vitro matured oocytes may increase pregnancy after intracytoplasmic sperm injection. Arch Androl 44, 197-205 (2000). 
10. Evsikov, A. V. \& Marín de Evsikova, C. Gene expression during the oocyte-to-embryo transition in mammals. Mol Reprod Dev 76, 805-818 (2009).

11. Fan, Y. et al. Aberrant expression of maternal Plk1 and Dctn3 results in the developmental failure of human in-vivo- and in-vitro-matured oocytes. Sci Rep 5, 8192 (2015).

12. Fan, X. et al. Single-cell RNA-seq transcriptome analysis of linear and circular RNAs in mouse preimplantation embryos. Genome Biol 16, 148 (2015).

13. Yanez, L. Z., Han, J., Behr, B. B., Pera, R. A. R. \& Camarillo, D. B. Human oocyte developmental potential is predicted by mechanical properties within hours after fertilization. Nat Commun 7 , 10809 (2016).

14. Assou, S. et al. The human cumulus--oocyte complex gene-expression profile. Hum Reprod 21, 1705-1719 (2006).

15. Jones, G. M. et al. Gene expression profiling of human oocytes following in vivo or in vitro maturation. Hum Reprod 23, 1138-1144 (2008).

16. Kocabas, A. M. et al. The transcriptome of human oocytes. Proc Natl Acad Sci U S A 103, $14027-14032$ (2006).

17. Xue, Z. et al. Genetic programs in human and mouse early embryos revealed by single-cell RNA sequencing. Nature 500, 593-597 (2013).

18. Zhang, Y. et al. Transcriptome Landscape of Human Folliculogenesis Reveals Oocyte and Granulosa Cell Interactions. Mol Cell 72, 1021-1034.e4 (2018).

19. Zhao, H. et al. Single-Cell Transcriptomics of Human Oocytes: Environment-Driven Metabolic Competition and Compensatory Mechanisms During Oocyte Maturation. Antioxid Redox Signal 30, 542-559 (2019).

20. Okuno, T. et al. Zygotic Nuclear F-Actin Safeguards Embryonic Development. Cell Rep 31, 107824 (2020). 
414 21. Messiaen, S. et al. Loss of the histone chaperone ASF1B reduces female reproductive capacity in $415 \quad$ mice. Reproduction 151, 477-489 (2016).

416 22. Li, Y. et al. Stella safeguards the oocyte methylome by preventing de novo methylation mediated by 417 DNMT1. Nature 564, 136-140 (2018).

23. Zhao, Z.-H. et al. RNA-Seq transcriptome reveals different molecular responses during human and mouse oocyte maturation and fertilization. BMC Genomics 21, 475 (2020).

24. Miettinen, H. E., Rayburn, H. \& Krieger, M. Abnormal lipoprotein metabolism and reversible female infertility in HDL receptor (SR-BI)-deficient mice. J Clin Invest 108, 1717-1722 (2001).

25. Simerly, C., Nowak, G., de Lanerolle, P. \& Schatten, G. Differential expression and functions of cortical myosin IIA and IIB isotypes during meiotic maturation, fertilization, and mitosis in mouse oocytes and embryos. Mol Biol Cell 9, 2509-2525 (1998).

26. Kalous, J., Kubelka, M., Solc, P., Susor, A. \& Motlík, J. AKT (protein kinase B) is implicated in meiotic maturation of porcine oocytes. Reproduction 138, 645-654 (2009).

27. Shimamoto, S. et al. Hypoxia induces the dormant state in oocytes through expression of Foxo3. Proc Natl Acad Sci U S A 116, 12321-12326 (2019).

28. Li, C. et al. The FSH-HIF-1 $\alpha$-VEGF Pathway Is Critical for Ovulation and Oocyte Health but Not Necessary for Follicular Growth in Mice. Endocrinology 161, (2020).

29. Zwaans, B. M. M. \& Lombard, D. B. Interplay between sirtuins, MYC and hypoxia-inducible factor in cancer-associated metabolic reprogramming. Dis Model Mech 7, 1023-1032 (2014).

30. Ma, R. et al. Sirt1/Nrf2 pathway is involved in oocyte aging by regulating Cyclin B1. Aging (Albany NY) 10, 2991-3004 (2018).

31. Chung, D. L. et al. Evidence that oocyte maturation induced by an oncogenic ras-p21 protein and insulin is mediated by overlapping yet distinct mechanisms. Exp Cell Res 203, 329-335 (1992). 
Selected Proteins, Histone Methylation Pattern and Spindle Integrity in Murine Oocytes. PLoS One 11, e0162722 (2016).

33. Liu, H. et al. miR-32-5p Regulates Lipid Accumulation in Intramuscular Fat of Erhualian Pigs by Suppressing KLF3. Lipids (2020) doi:10.1002/lipd.12294.

34. Dunning, K. R., Russell, D. L. \& Robker, R. L. Lipids and oocyte developmental competence: the role of fatty acids and $\beta$-oxidation. Reproduction 148, R15-27 (2014).

35. Aizawa, R. et al. Synthesis and maintenance of lipid droplets are essential for mouse preimplantation embryonic development. Development 146, dev181925 (2019).

36. Wang, H. et al. Roles of Tet2 in meiosis, fertility and reproductive aging. Protein Cell (2020) doi:10.1007/s13238-020-00805-8.

37. Romasko, E. J., Amarnath, D., Midic, U. \& Latham, K. E. Association of maternal mRNA and phosphorylated EIF4EBP1 variants with the spindle in mouse oocytes: localized translational control supporting female meiosis in mammals. Genetics 195, 349-358 (2013).

38. $\mathrm{Su}, \mathrm{Y}$.-Q. et al. Oocyte regulation of metabolic cooperativity between mouse cumulus cells and oocytes: BMP15 and GDF9 control cholesterol biosynthesis in cumulus cells. Development 135, $111-121$ (2007).

39. Awe, J. P. \& Byrne, J. A. Identifying candidate oocyte reprogramming factors using cross-species global transcriptional analysis. Cell Reprogram 15, 126-133 (2013).

40. Farese, R. V., Ruland, S. L., Flynn, L. M., Stokowski, R. P. \& Young, S. G. Knockout of the mouse apolipoprotein B gene results in embryonic lethality in homozygotes and protection against diet-induced hypercholesterolemia in heterozygotes. Proceedings of the National Academy of Sciences 92, 1774-1778 (1995).

41. Scalici, E. et al. Apolipoprotein B is regulated by gonadotropins and constitutes a predictive biomarker of IVF outcomes. Reprod Biol Endocrinol 14, 28 (2016). 
42. Dominguez, F. et al. Comparative protein-profile analysis of implanted versus non-implanted human blastocysts. Human Reproduction 23, 1993-2000 (2008).

43. Bolger, A. M., Lohse, M. \& Usadel, B. Trimmomatic: a flexible trimmer for Illumina sequence data. Bioinformatics 30, 2114-2120 (2014).

44. Martin, M. Cutadapt removes adapter sequences from high-throughput sequencing reads. EMBnet j. 17, $10(2011)$.

45. Dobin, A. et al. STAR: ultrafast universal RNA-seq aligner. Bioinformatics 29, 15-21 (2013).

46. Liao, Y., Smyth, G. K. \& Shi, W. featureCounts: an efficient general purpose program for assigning sequence reads to genomic features. Bioinformatics 30, 923-930 (2014).

47. Robinson, J. T. et al. Integrative genomics viewer. Nat Biotechnol 29, 24-26 (2011).

48. Love, M. I., Huber, W. \& Anders, S. Moderated estimation of fold change and dispersion for RNA-seq data with DESeq2. Genome Biol 15, 550 (2014).

49. Huang, D. W., Sherman, B. T. \& Lempicki, R. A. Systematic and integrative analysis of large gene lists using DAVID bioinformatics resources. Nat Protoc 4, 44-57 (2009).

50. Huang, D. W., Sherman, B. T. \& Lempicki, R. A. Bioinformatics enrichment tools: paths toward the comprehensive functional analysis of large gene lists. Nucleic Acids Res 37, 1-13 (2009).

51. Babicki, S. et al. Heatmapper: web-enabled heat mapping for all. Nucleic Acids Res 44, W147-153 (2016). 


\section{Acknowledgements}

We thank K.K. DNAFORM (Yokohama, Japan) for RNA-seq analyses. We thank Ms N Backes Kamimura for proof reading. This research was supported by JSPS KAKENHI Grant Numbers JP17H05045, JP19H05271, JP19H05751, JP20K21376 to K.Mi., by The Naito Foundation to K.Mi., by Takeda Science Foundation to K.Mi, by a Kindai University Research Grant (19-II-1) to K.Mi., and by Young Researcher Support Project of Mie University and a Start-up Joint Research Promotion Project of Mie University with small- and medium-sized enterprises.

\section{Author contributions}

K.Mi. conceived the project. H.T. and K.Mi. designed experiments. H.T., M.Y., M.F., and K.Mi. performed experiments. M.Y. and K.Mi. analyzed data. K.Ma. provided an analytic tool. T.M., M.N., E. K., T.I., and K.Mi. supervised research. K.Mi, mainly wrote the manuscript. H.T., and M.Y. helped writing the manuscript.

\section{Competing interests}

The authors declare no competing financial interests.

\section{Additional information}

Supplemental information is available for this paper. 

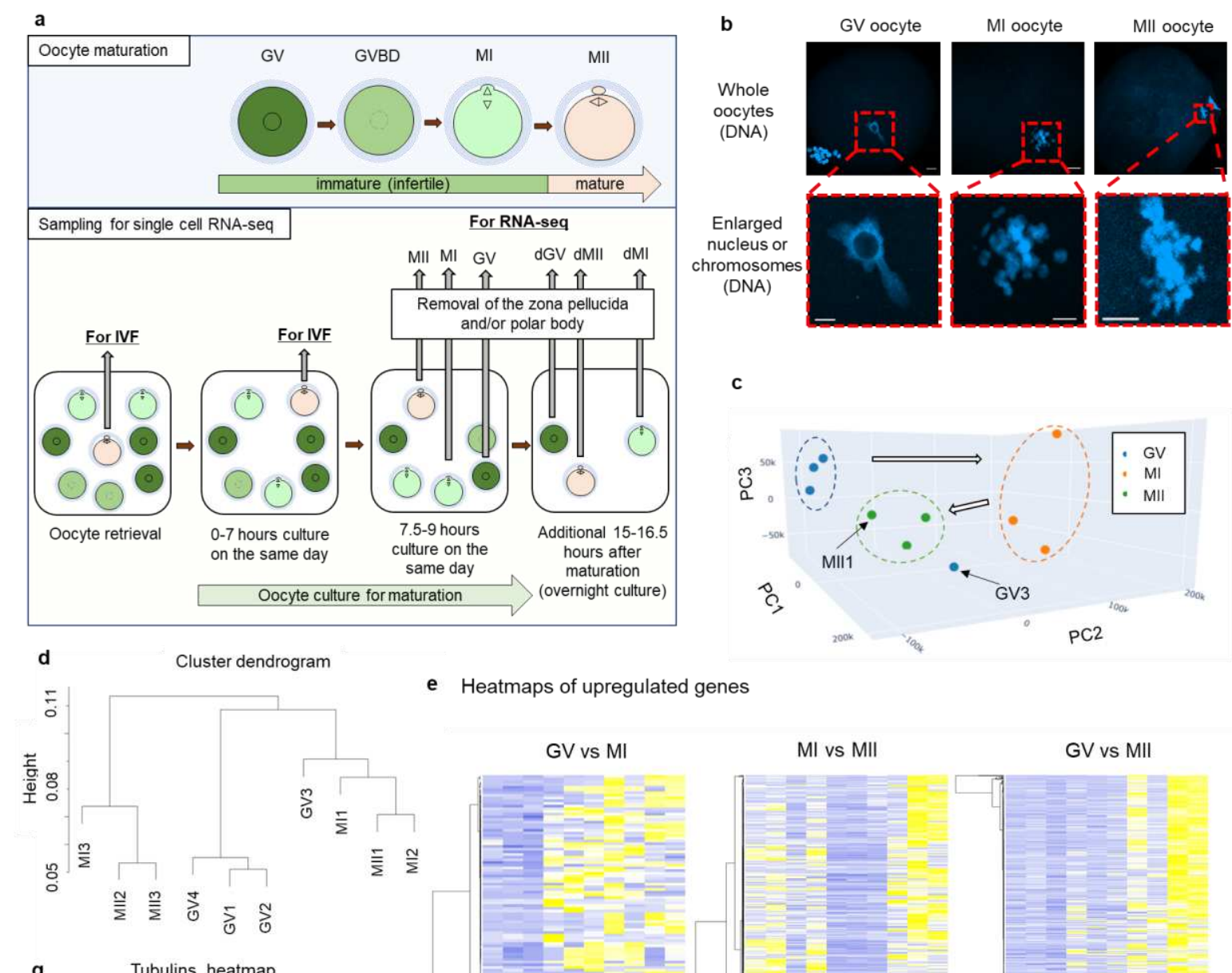

g Tubulins_heatmap
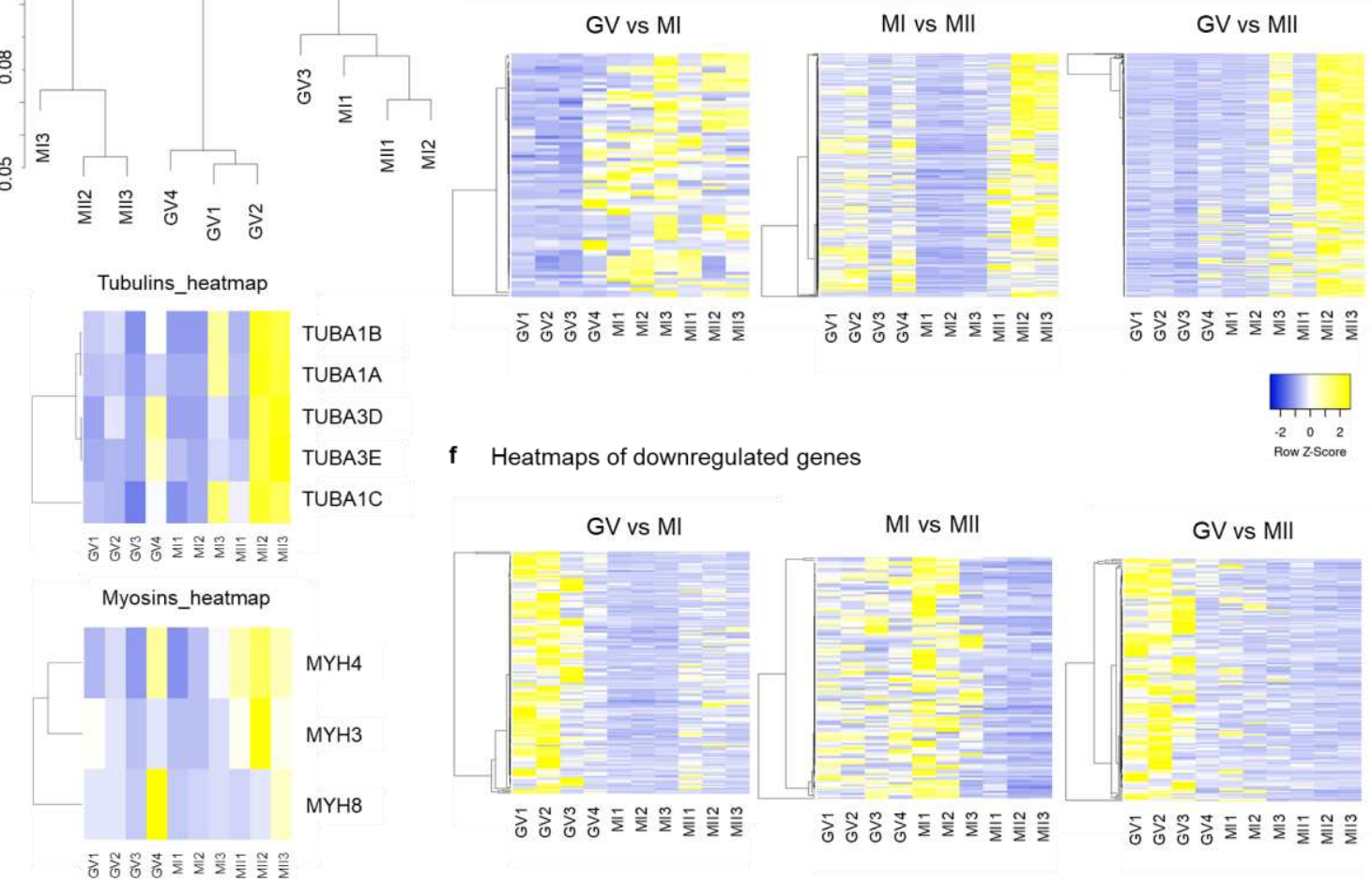

506 Fig. 1. Transcriptomic changes of human oocytes during in vitro maturation. a A schematic

507 diagram of human oocyte retrieval for single cell RNA-seq analyses. Ten oocytes were collected 
508 from 7 patients. b Representative images of human oocytes at different maturation stages. The bottom

509 panels show higher magnification images of the regions indicated in the top panels by dashed boxes.

510 DNA was stained by DAPI. Scale bars represent $20 \mu \mathrm{m}$ for whole oocyte images and $10 \mu \mathrm{m}$ for

511 enlarged images. $\mathbf{c}$ PCA of the gene expression profiles (3-4 biological replicates per each maturation

512 stage). Blue dots: GV samples, Orange dots: MI samples, and Green dots: MII samples. d Hierarchical

513 clustering dendrogram generated using the gene expression profiles of human oocytes at different

514 maturation stages. Spearman correlation was used. e, f Heatmaps depicting the gene expression levels of

515 DEGs identified in the indicated comparisons. Color key with z-score is shown. (e) shows upregulated

516 genes in MI (GV vs MI), MII (MI vs MII), and MII (GV vs MII) samples, while (f) indicates

517 downregulated genes in the same comparisons. $\mathrm{g}$ Heatmaps depicting the gene expression levels of

518 Tubulin-related and myosin-related genes. GV; oocytes at the germinal vesicle stage, MI; maturating

519 oocytes at the metaphase I stage, MII; oocytes at the metaphase II stage.

520 
a
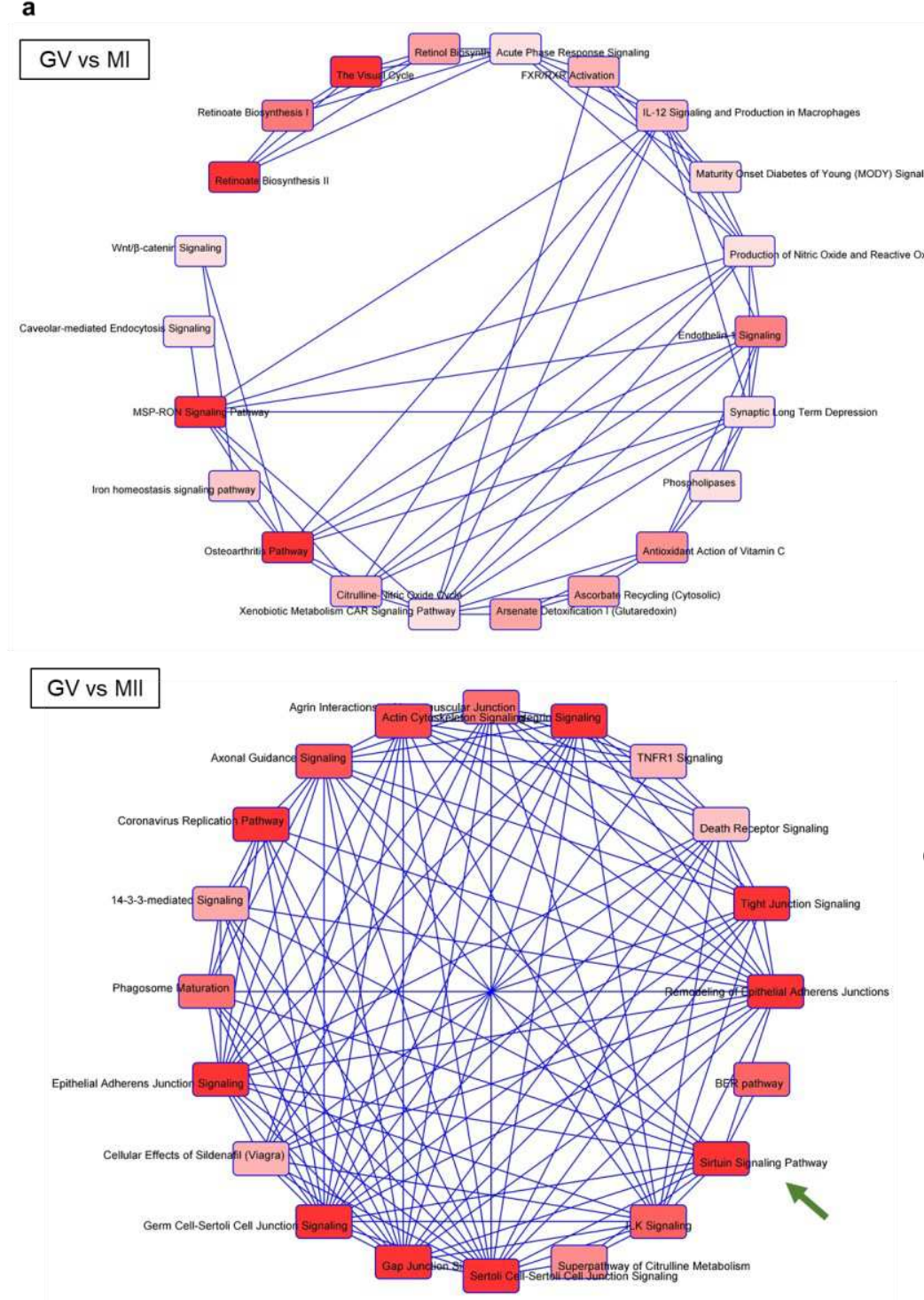

b
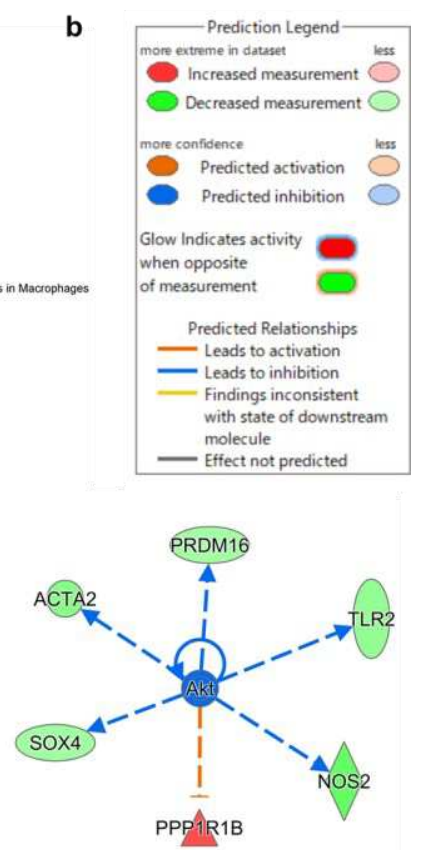

c

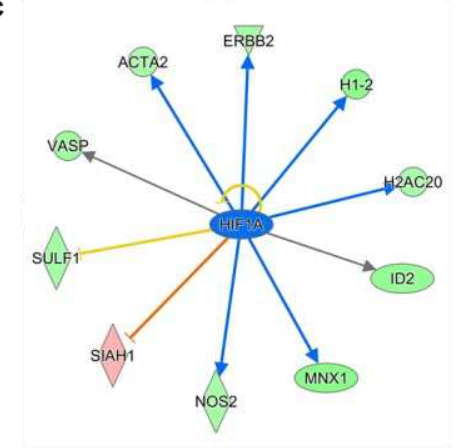

Fig. 2. Gene regulatory networks that are associated with dynamic transcriptome changes during

523 human oocyte maturation. a Canonical pathways predicted by IPA using the DEG list of GV vs MI or

524 GV vs MII. Significant terms are indicated in red colors: darker the color more significant the term is $(P$

$525<0.05$, Fisher's exact test). Closely related terms are connected to each other. A green arrow indicates

526 Sirtuin signaling pathway as explained in the relevant text. b Downstream targets of AKT were found in the DEG list by IPA. Gene regulatory networks of AKT in human oocytes were predicted. $\mathbf{c}$ Downstream 
528 targets of HIF1A were found in the DEG list by IPA. Blue arrows mean 'Leads to inhibition', orange

529 arrows mean 'Leads to activation', a yellow arrow means 'Inconsistent with state of downstream

530 molecules', black arrows mean 'Effect not predicted'. Molecules colored green, red and blue show 531 'downregulated', 'upregulated', and 'predicted inhibition', respectively.

532

533 
a
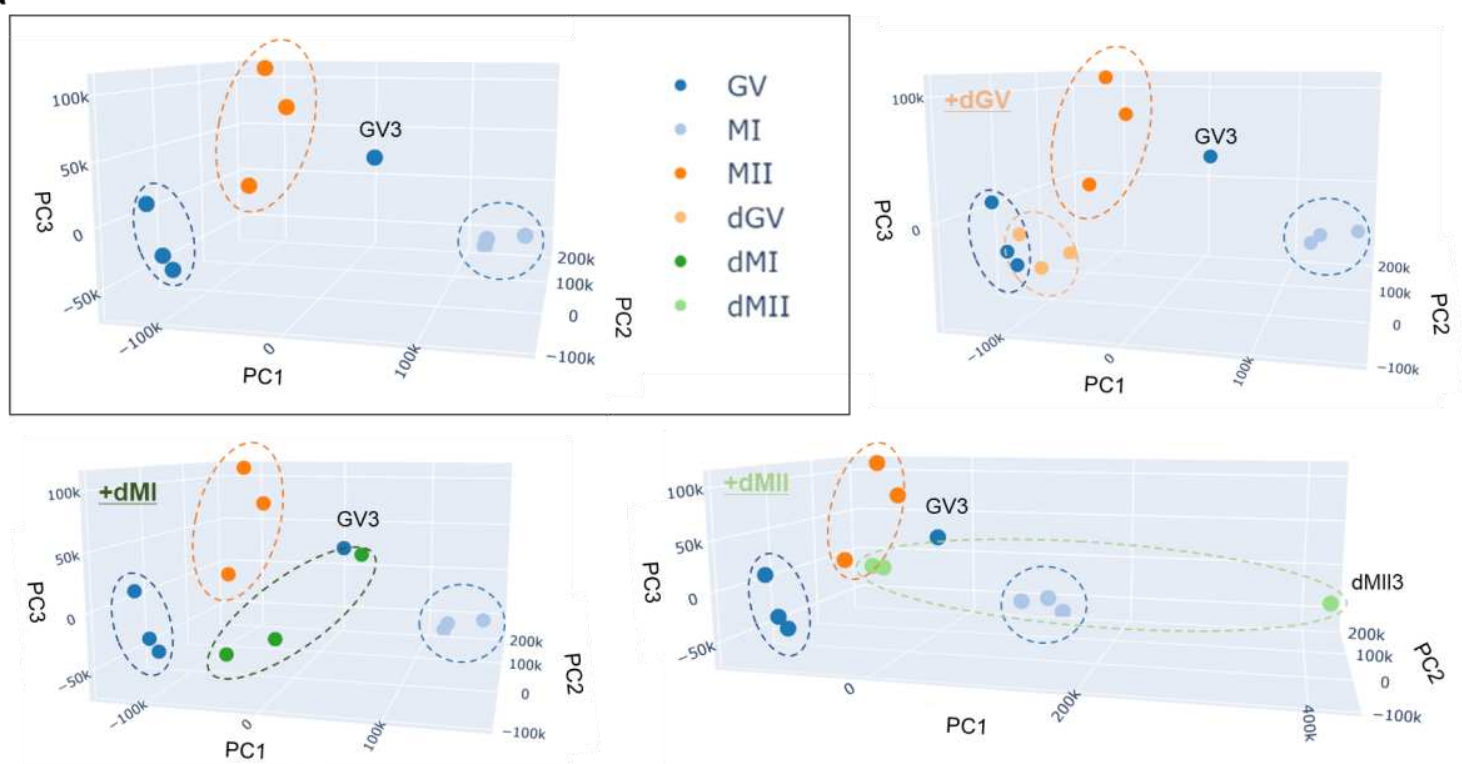

b Heatmaps of upregulated genes GV vs dGV

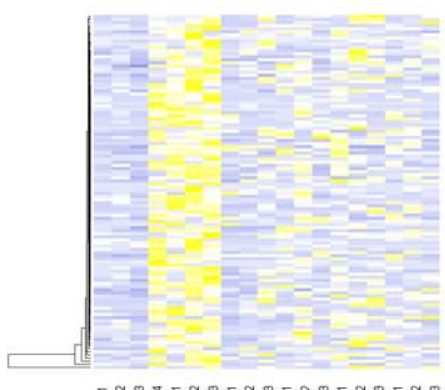

Ml vs $\mathrm{dMI}$
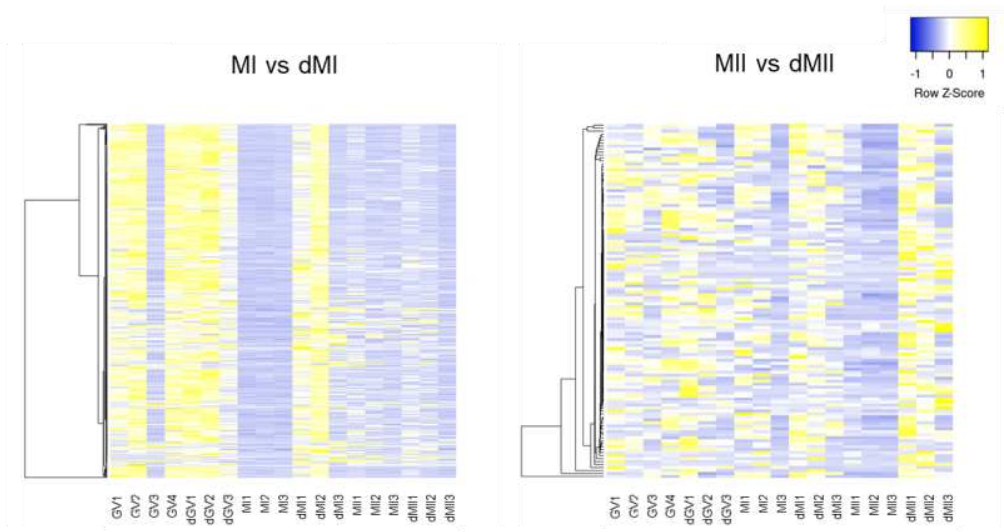

C Heatmaps of downregulated genes

GV vs dGV

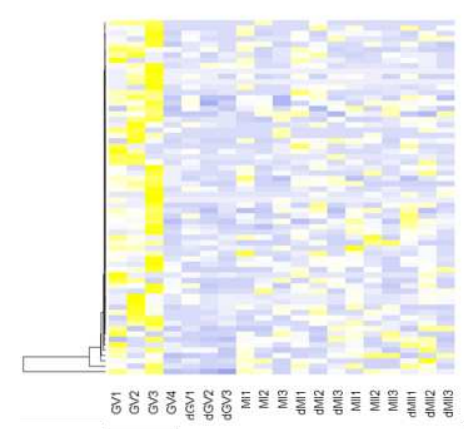

MI vs $d M I$

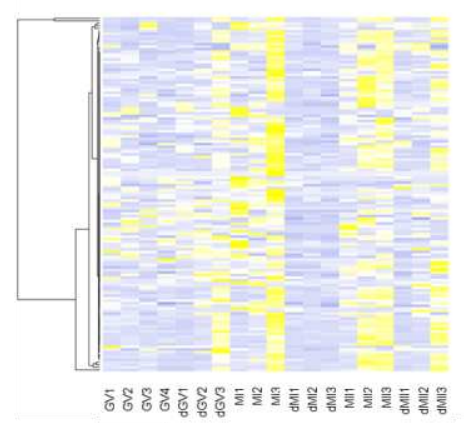

MII vs dMII

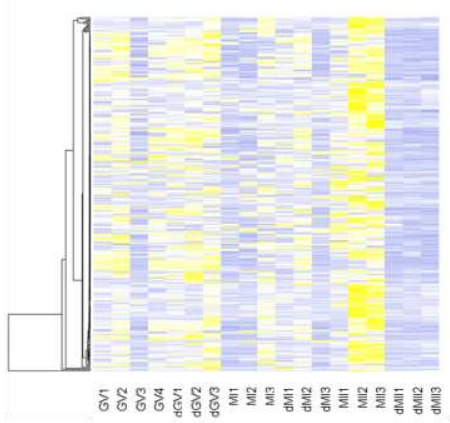

Fig. 3. Human oocytes that are delayed in their maturation show different transcriptomes from 
536 those matured in an earlier timing. a PCA of the gene expression profiles (3-4 biological replicates per

537 each category). The top left image in the rectangle includes samples collected on the day for oocyte

538 retrieval (Fig. 1a) (GV, MI and MII). Nine delayed oocytes were collected from 7 patients. Blue dots:

539 GV samples, light blue dots: MI samples, dark orange dots: MII samples, light orange dots: dGV

540 samples, dark green dots: dMI samples, and light green dots: dMII samples. b, c Heatmaps depicting the

541 gene expression levels of DEGs identified in the indicated comparisons. (b) shows upregulated genes in

$542 \mathrm{dGV}(\mathrm{GV}$ vs dGV), dMI (MI vs dMI), and dMII (MII vs dMII) samples, while (c) indicates

543 downregulated genes in the same comparisons. Color key with z-score is shown. dGV; oocytes that were

544 at the germinal vesicle stage the day after oocyte retrieval, dMI; oocytes that were at the metaphase I

545 stage the day after oocyte retrieval, dMII; oocytes that were at the metaphase II stage the day after 546 oocyte retrieval.

547

548 
a

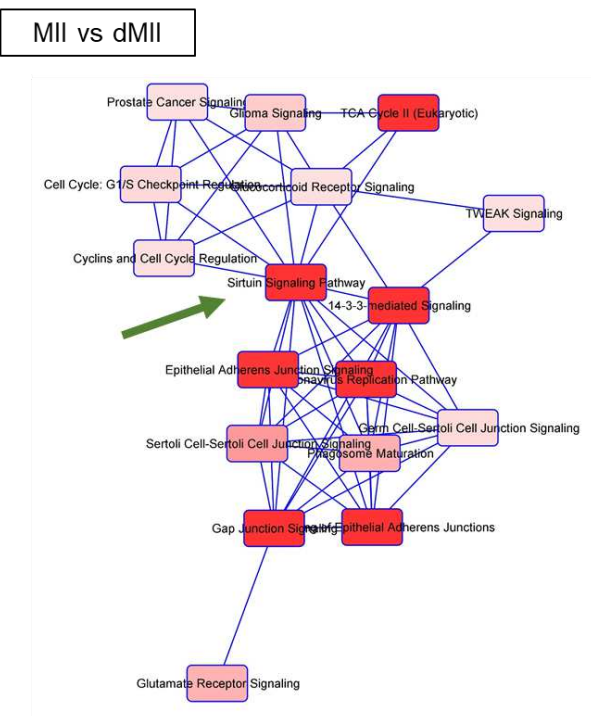

b

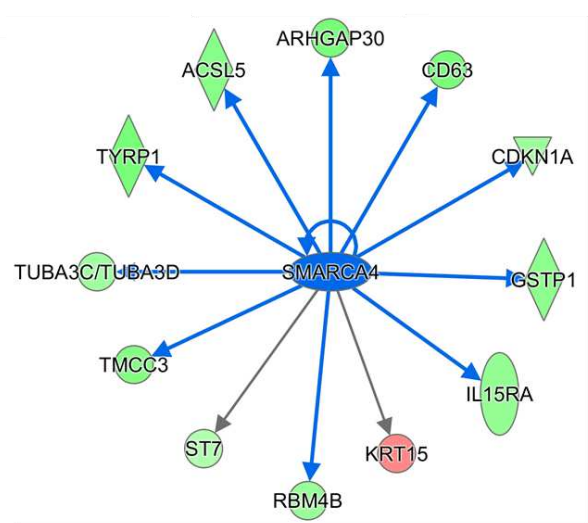
and 'predicted inhibition', respectively.

Fig. 4. Gene regulatory networks are altered in the human oocytes that are delayed in their maturation. a Canonical pathways predicted by IPA using the DEG list of MII vs dMII. Significant terms are indicated in red colors: darker the color more significant the term is $(P<0.05$, Fisher's exact test). Closely related terms are connected to each other. A green arrow indicates Sirtuin signaling pathway as explained in the relevant text. b Downstream targets of SMARCA4 were found in the DEG list by IPA. Gene regulatory networks of SMARCA4 in human oocytes were predicted. SMARCA4 affects molecules indicated at the end of arrows, blue arrows mean 'Leads to inhibition', black arrows mean 'Effect not predicted'. Molecules colored green, red and blue show 'downregulated', 'upregulated', 
a

Heatmaps of upregulated genes

MII1 vs MII2

MII2 vs MII3

MII3 vs MII1
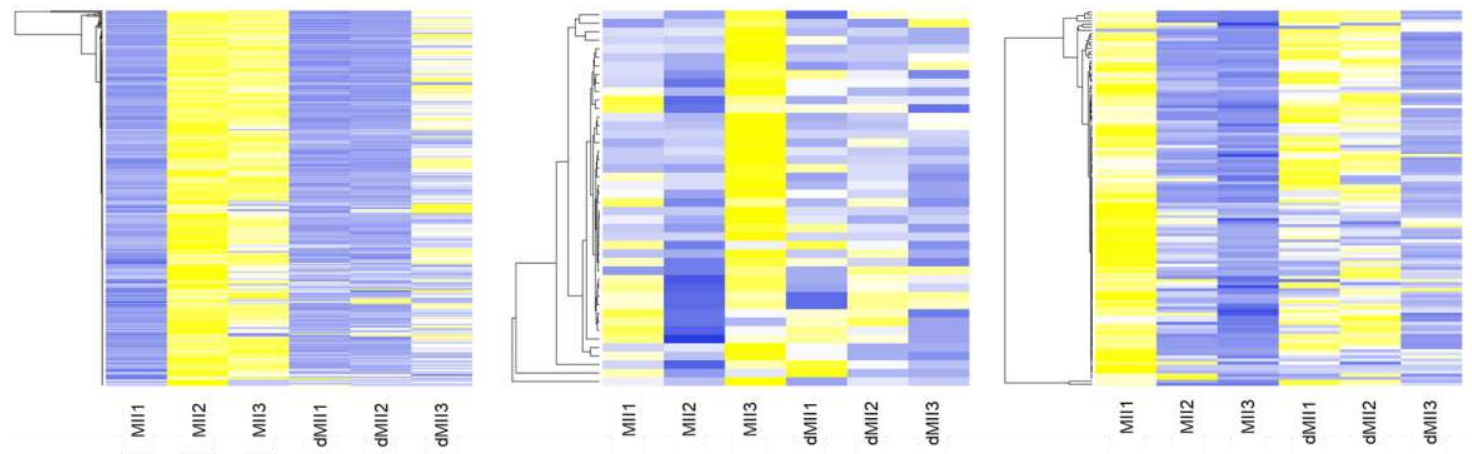

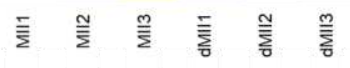

b Heatmaps of downregulated genes MII1 vs MII2

MII2 vs MII3

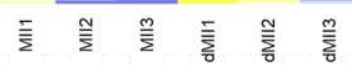

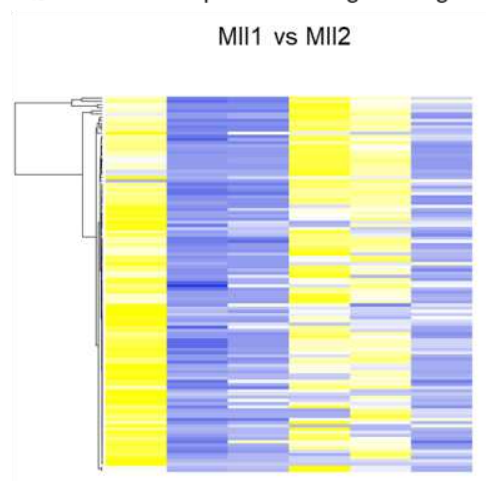

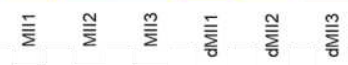
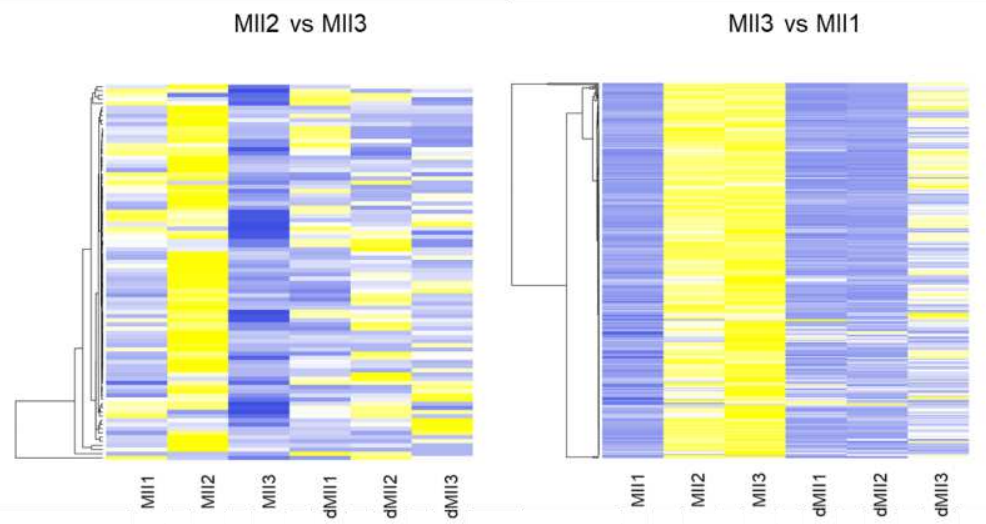

C
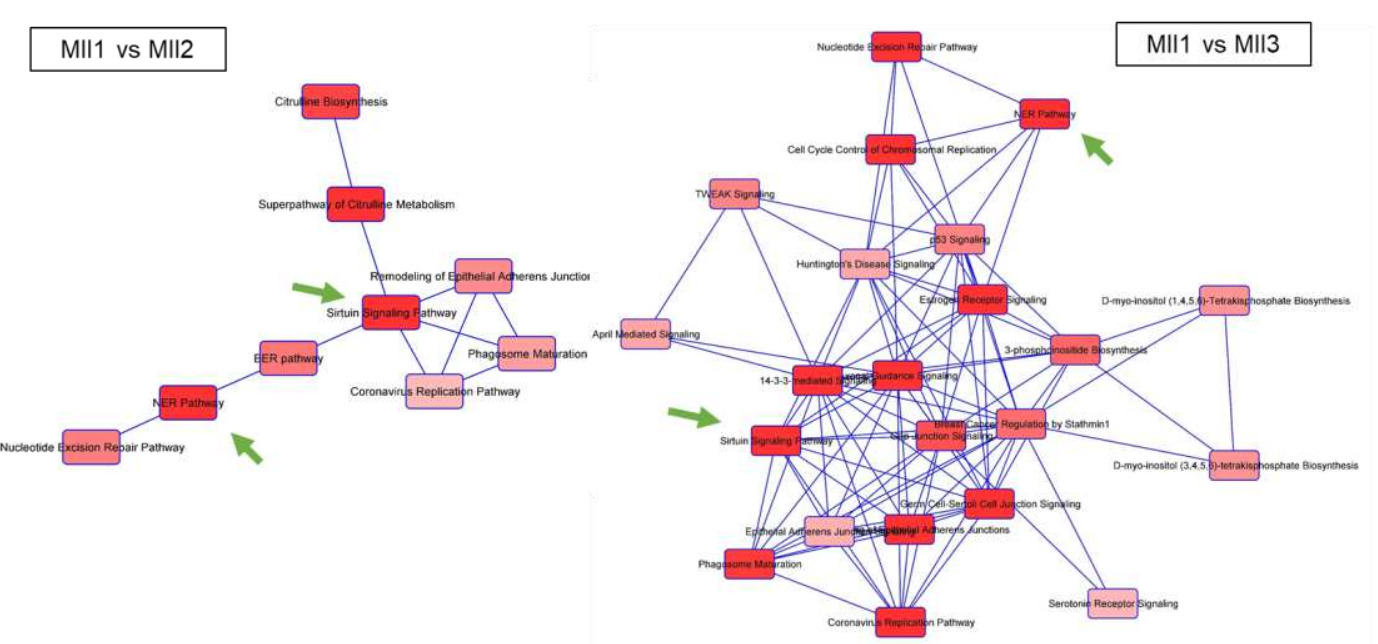

Fig. 5. Gene regulatory networks that are altered in different patients. a, b Heatmaps depicting the gene expression levels of DEGs identified in the indicated comparisons. (a) shows upregulated genes in MII2 (MII1 vs MII2), MII3 (MII2 vs MII3), and MII1 (MII3 vs MII1) samples, while (b) indicates 
565 downregulated genes in the same comparisons. Color key with z-score is shown. c Canonical pathways 566 predicted by IPA using the DEG list of MII1 vs MII2 or MII1 vs MII3. Significant terms are indicated in 567 red colors: darker the color more significant the term is $(P<0.05$, Fisher's exact test). Closely related 568 terms are connected to each other. Green arrows indicate commonly misregulated pathways.

569

570

571 
a

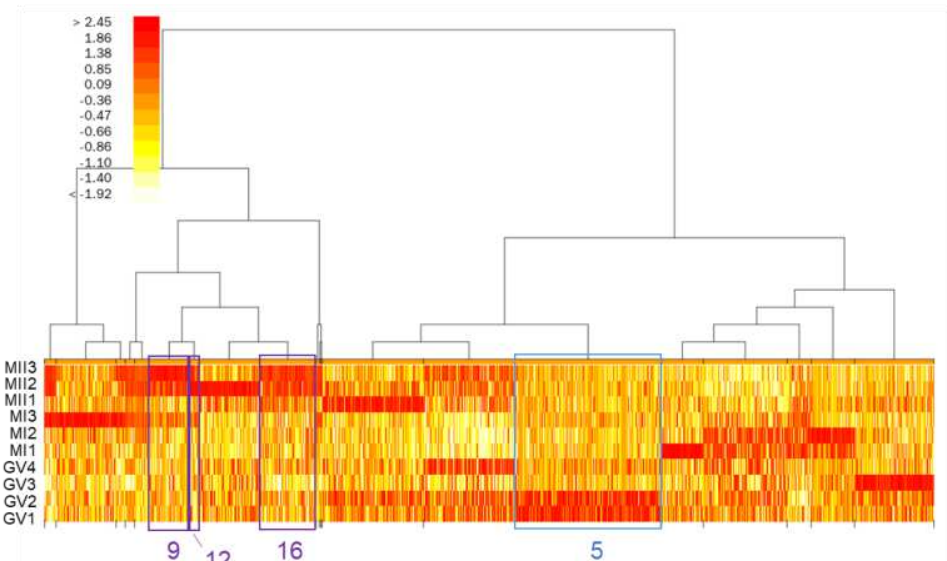

b
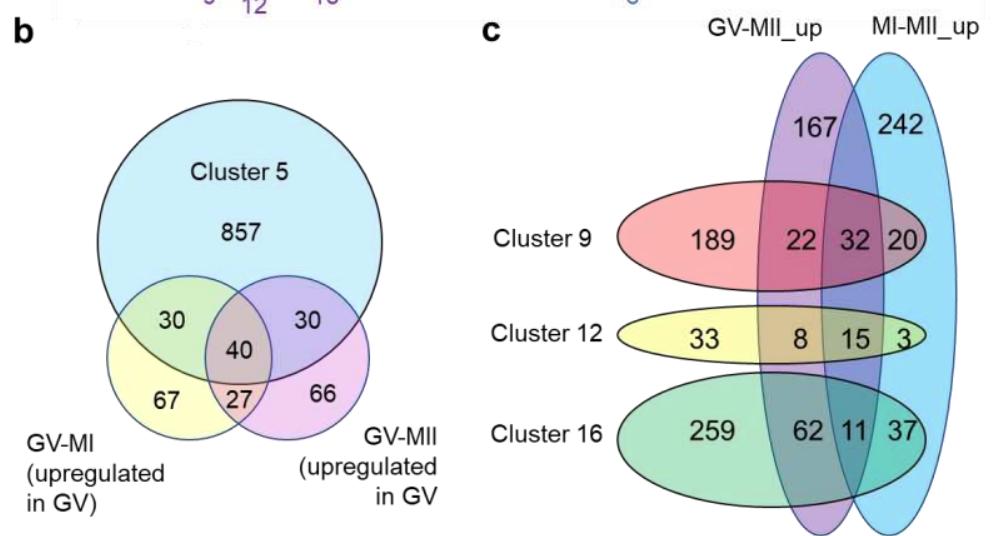

d

572

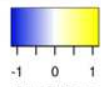

GV
GV
MII
MII
MIII
MIII
dMII
dMI
dMI

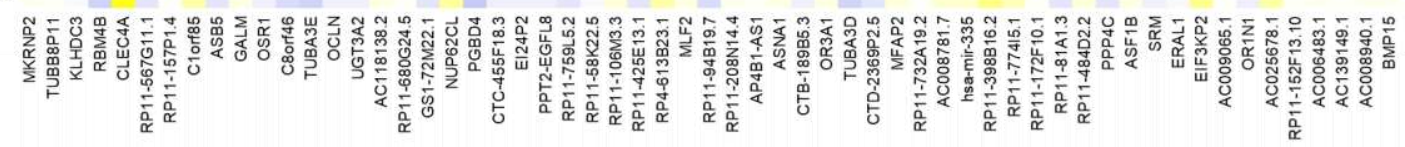

573 Fig. 6. Marker genes for human GV and MII oocytes and potential biomarkers for MII oocytes

574 with high quality. a A heatmap of gene expression levels after clustering based on expression patterns. 
575 Twenty clusters were generated and clusters 5, 9, 12, and 16 are indicated. Color key indicates

$576 \log 2 \mathrm{FC}($ FoldChange). b Venn diagrams showing the numbers of total and overlapping genes among

577 genes that belong to cluster 5, upregulated genes in the GV vs MI comparison, and upregulated genes in

578 the GV vs MII comparison. $\mathbf{c}$ Venn diagrams showing the numbers of total and overlapping genes among

579 genes that belong to clusters 9, 12, and 16, upregulated genes in the GV vs MII comparison, and

580 upregulated genes in the MI vs MII comparison. d A heatmap depicting the gene expression levels of

581 candidate genes for human MII oocytes with high quality. Gene symbols are listed. Color key with

582 z-score is shown.

583

584 
Figures
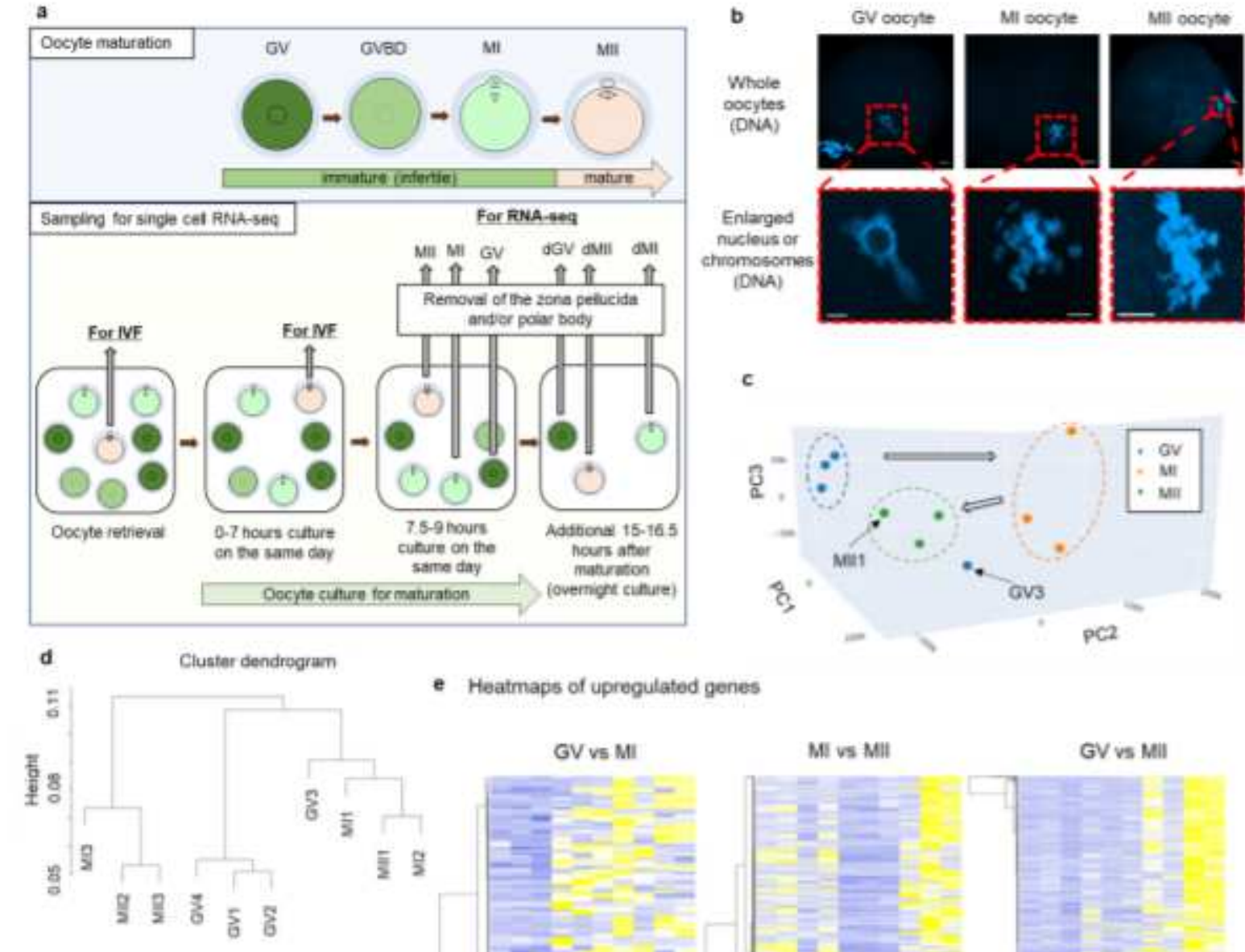

g
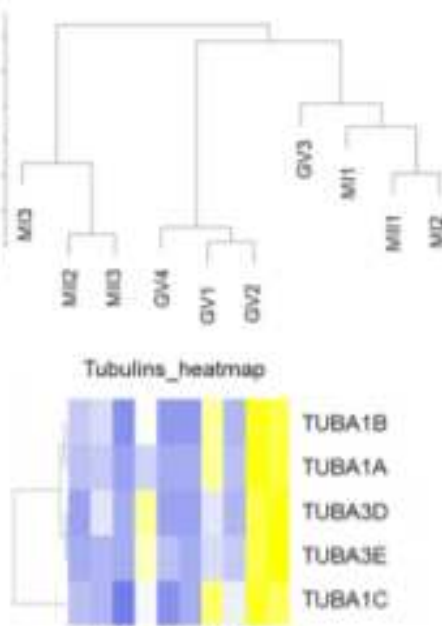

e Heatmaps of upregulated genes
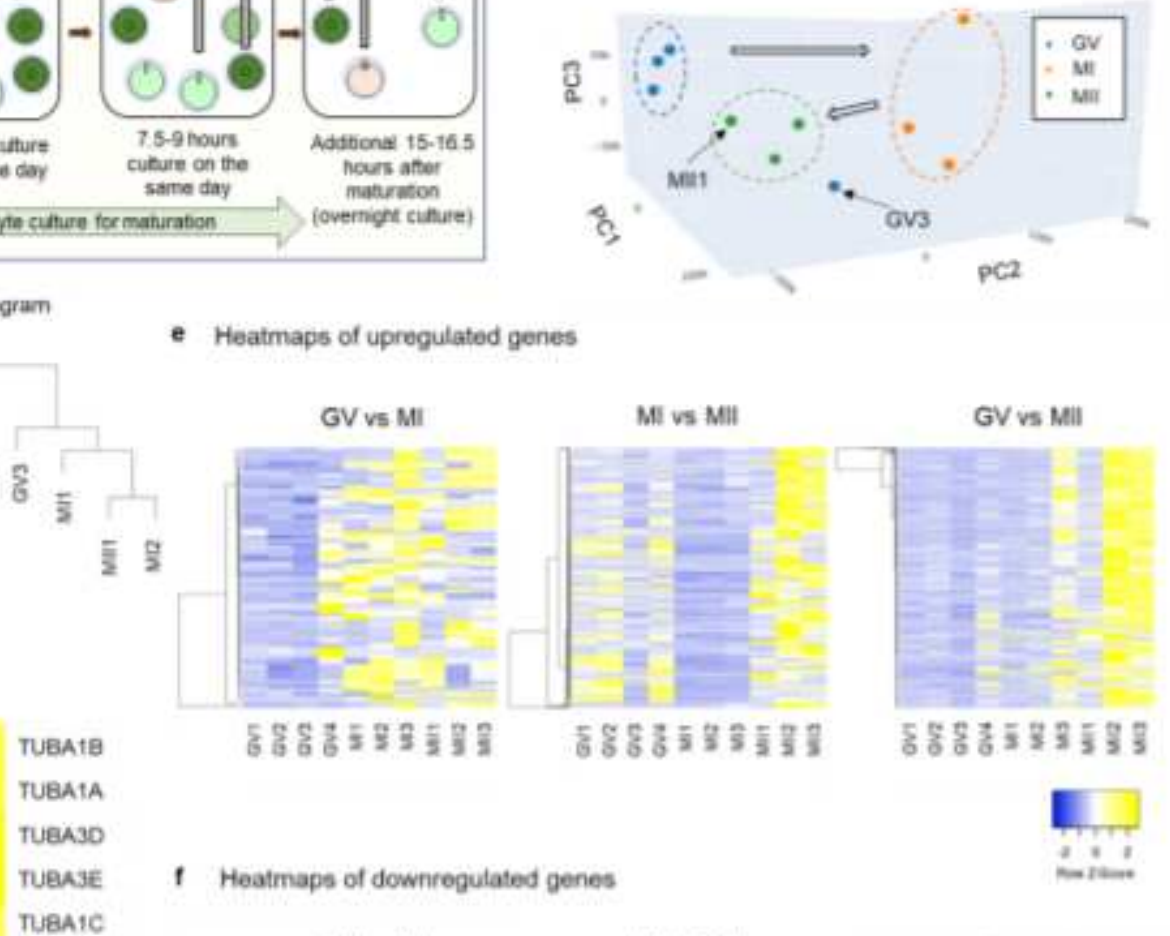

MII vs MIII

GV vs Mil
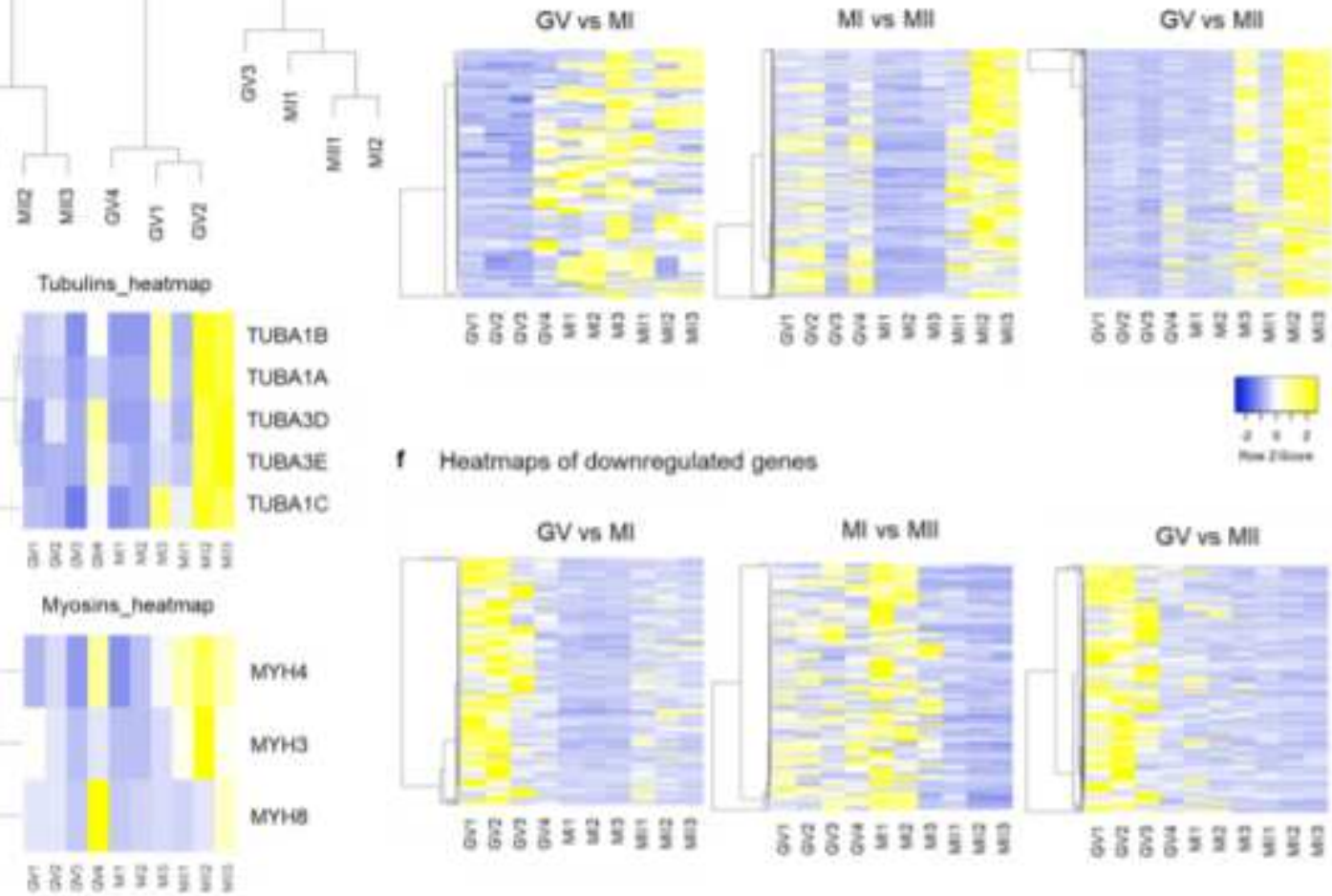

GV vs Mi

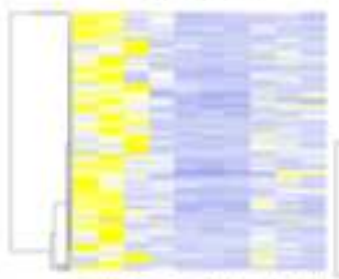

Mi vs Mili
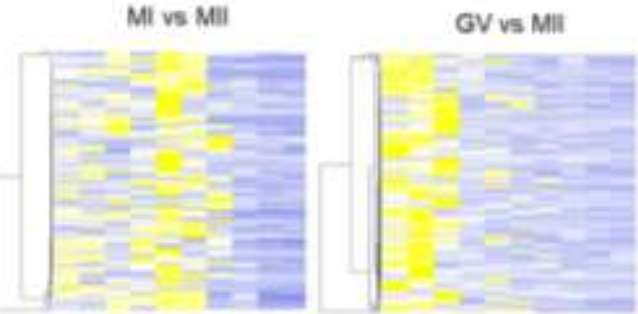

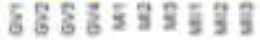

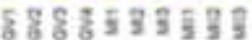

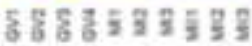

\section{Figure 1}

Transcriptomic changes of human oocytes during in vitro maturation. a A schematic diagram of human oocyte retrieval for single cell RNA-seq analyses. Ten oocytes were collected from 7 patients. $b$ Representative images of human oocytes at different maturation stages. The bottom panels show higher magnification images of the regions indicated in the top panels by dashed boxes. DNA was stained by 
DAPI. Scale bars represent $20 \mu \mathrm{m}$ for whole oocyte images and $10 \mu \mathrm{m}$ for enlarged images. c PCA of the gene expression profiles (3-4 biological replicates per each maturation stage). Blue dots: GV samples, Orange dots: MI samples, and Green dots: MII samples. d Hierarchical clustering dendrogram generated using the gene expression profiles of human oocytes at different maturation stages. Spearman correlation was used. e, $f$ Heatmaps depicting the gene expression levels of DEGs identified in the indicated comparisons. Color key with z-score is shown. (e) shows upregulated genes in MI (GV vs MI), MII (MI vs MII), and MII (GV vs MII) samples, while (f) indicates downregulated genes in the same comparisons. g Heatmaps depicting the gene expression levels of Tubulin-related and myosin-related genes. GV; oocytes at the germinal vesicle stage, Ml; maturating oocytes at the metaphase I stage, MII; oocytes at the metaphase II stage.

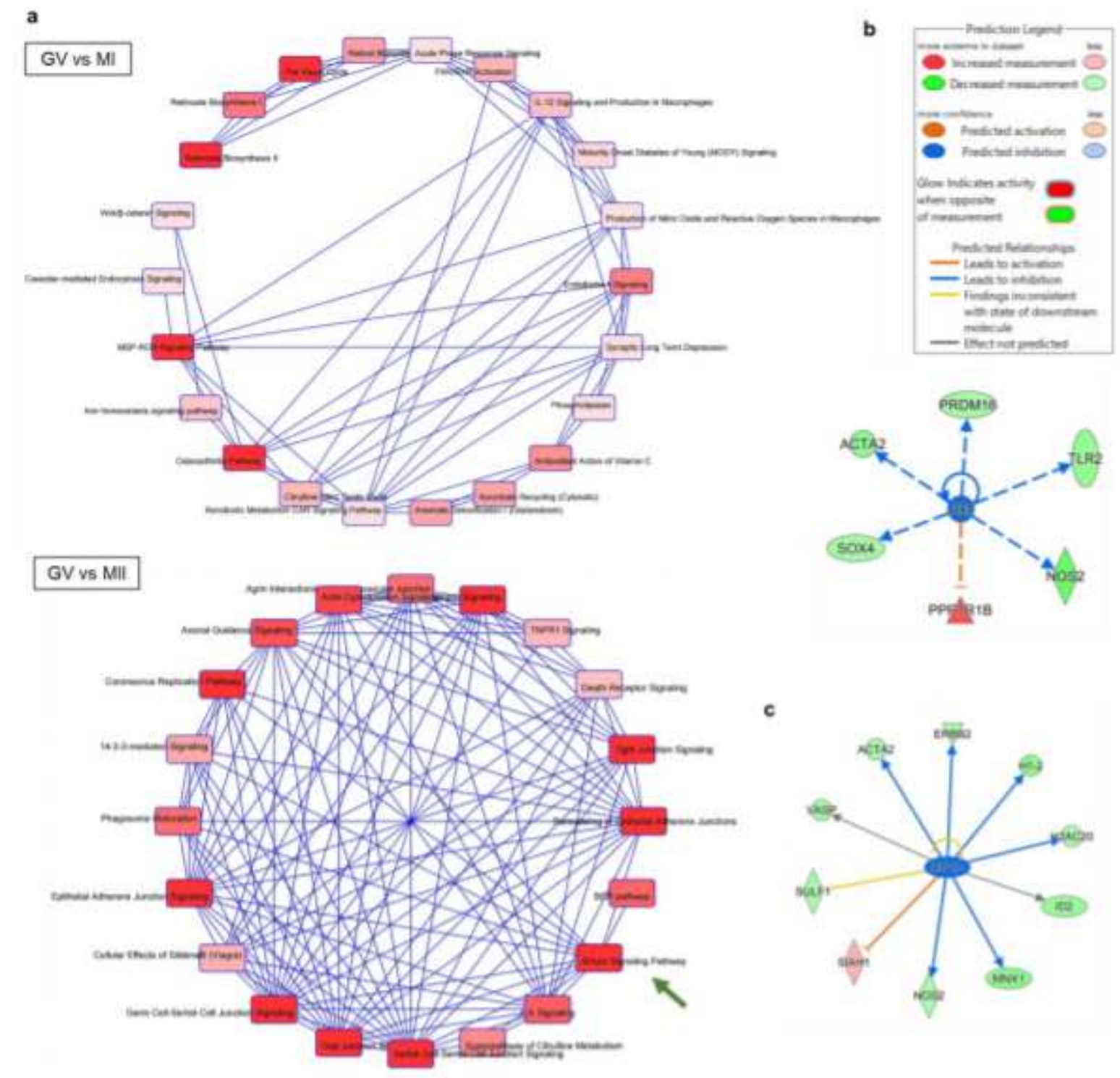

\section{Figure 2}

Gene regulatory networks that are associated with dynamic transcriptome changes during human oocyte maturation. a Canonical pathways predicted by IPA using the DEG list of GV vs MI or GV vs MII. Significant terms are indicated in red colors: darker the color more significant the term is $(P<0.05$, 
Fisher's exact test). Closely related terms are connected to each other. A green arrow indicates Sirtuin signaling pathway as explained in the relevant text. b Downstream targets of AKT were found in the DEG list by IPA. Gene regulatory networks of AKT in human oocytes were predicted. c Downstream targets of HIF1A were found in the DEG list by IPA. Blue arrows mean 'Leads to inhibition', orange arrows mean 'Leads to activation', a yellow arrow means 'Inconsistent with state of downstream molecules', black arrows mean 'Effect not predicted'. Molecules colored green, red and blue show 'downregulated', 'upregulated', and 'predicted inhibition', respectively.

a
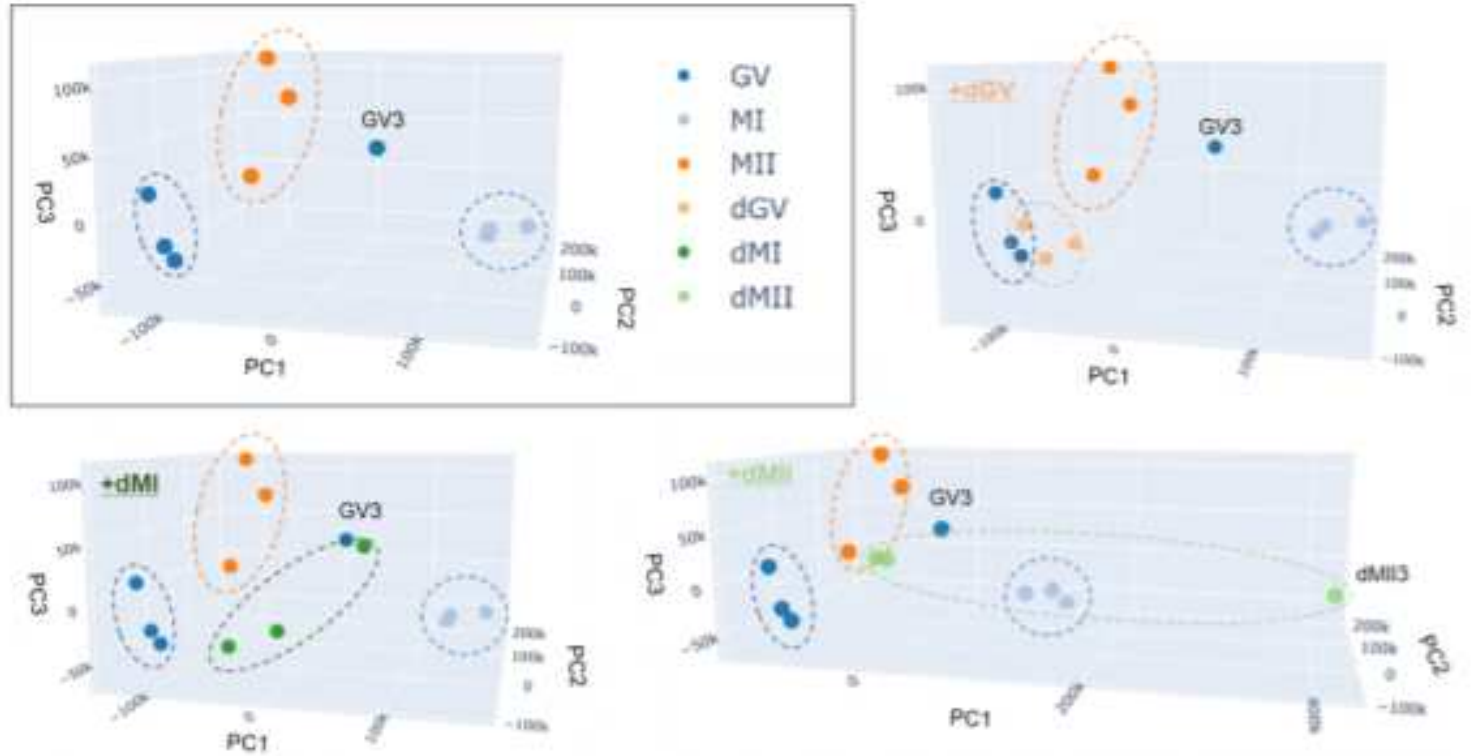

b Heatmaps of upregulated genes

GV vs dGV

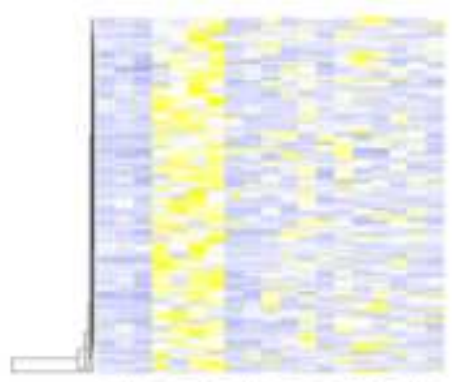

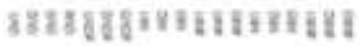

c

Heatmaps of downergulated gones

GV vi dGV

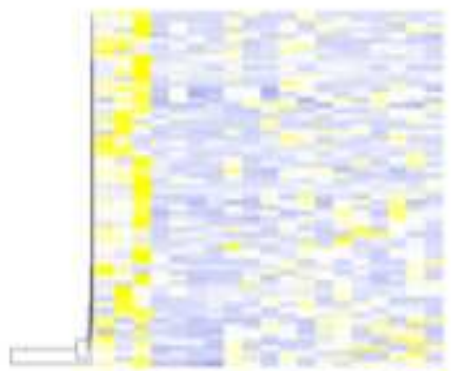

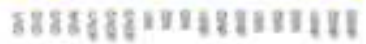

Mi vs dMI

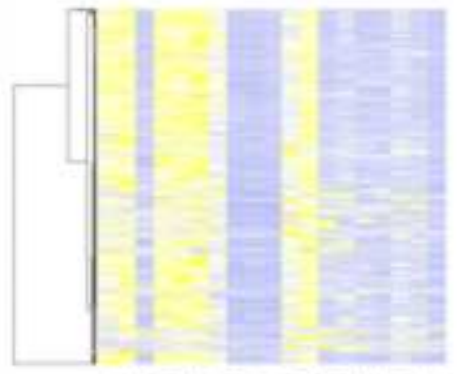

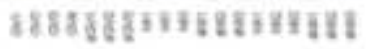

Mit vs dMI

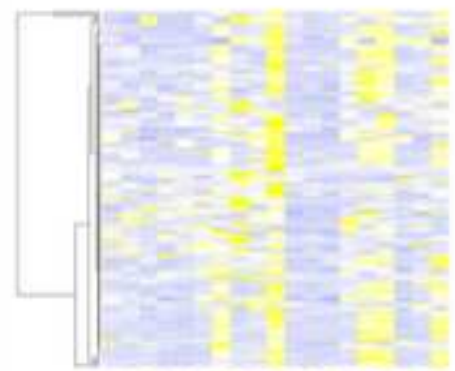

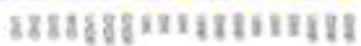

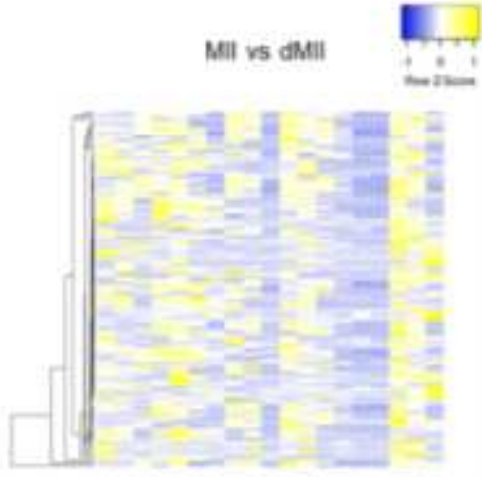

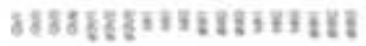

Mil vs dMell

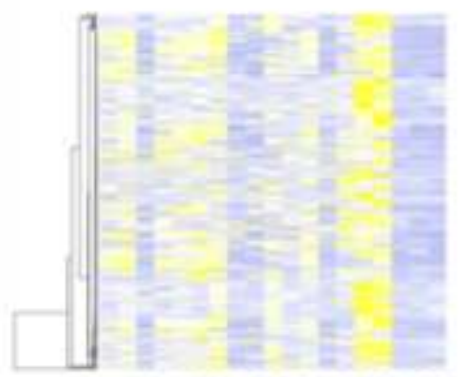

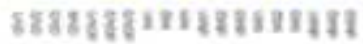




\section{Figure 3}

Human oocytes that are delayed in their maturation show different transcriptomes from those matured in an earlier timing. a PCA of the gene expression profiles (3-4 biological replicates per each category). The top left image in the rectangle includes samples collected on the day for oocyte retrieval (Fig. 1a) (GV, MI and MII). Nine delayed oocytes were collected from 7 patients. Blue dots: GV samples, light blue dots: MI samples, dark orange dots: MII samples, light orange dots: dGV samples, dark green dots: dMI samples, and light green dots: dMII samples. b, c Heatmaps depicting the gene expression levels of DEGs identified in the indicated comparisons. (b) shows upregulated genes in dGV (GV vs dGV), dMI (MI vs dMI), and dMII (MII vs dMII) samples, while (c) indicates downregulated genes in the same comparisons. Color key with z-score is shown. dGV; oocytes that were at the germinal vesicle stage the day after oocyte retrieval, $\mathrm{dMl}$; oocytes that were at the metaphase I 545 stage the day after oocyte retrieval, dMIl; oocytes that were at the metaphase II stage the day after ocyte retrieval.

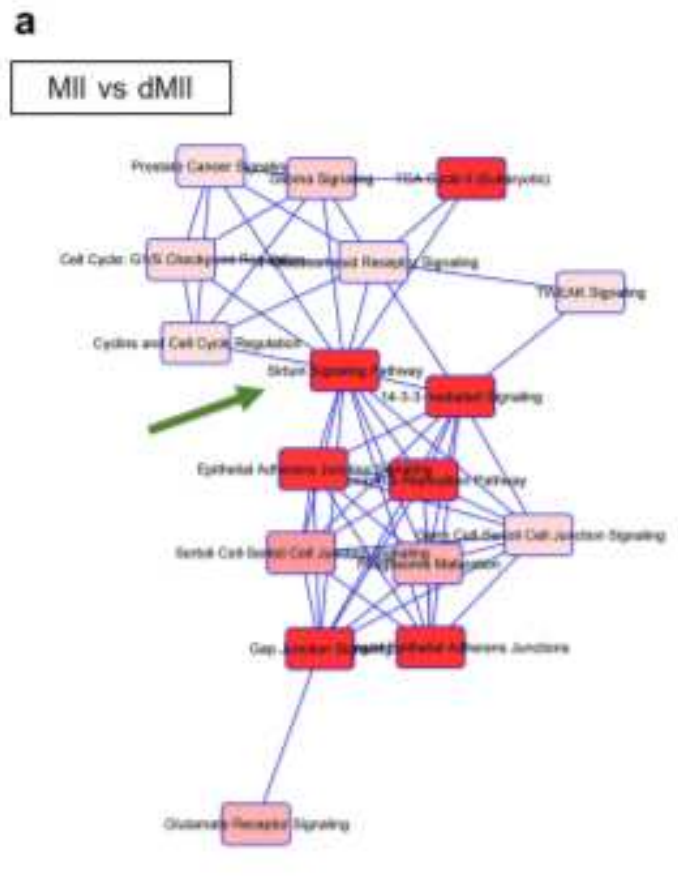

b
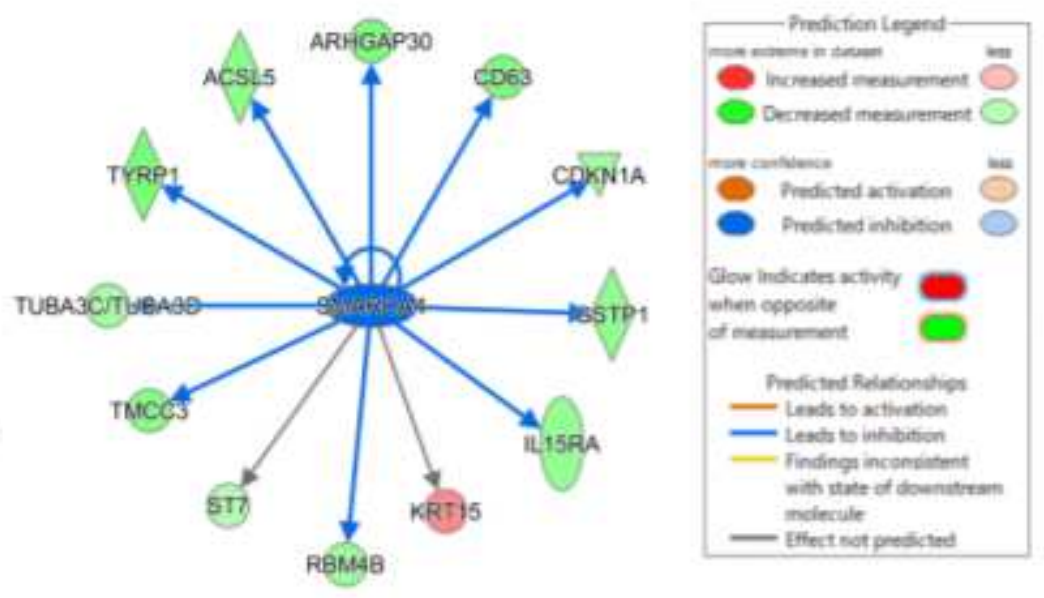

\section{Figure 4}

Gene regulatory networks are altered in the human oocytes that are delayed in their maturation. a Canonical pathways predicted by IPA using the DEG list of MII vs dMII. Significant terms are indicated in red colors: darker the color more significant the term is $(P<0.05$, Fisher's exact test). Closely related terms are connected to each other. A green arrow indicates Sirtuin signaling pathway as explained in the relevant text. b Downstream targets of SMARCA4 were found in the DEG list by IPA. Gene regulatory networks of SMARCA4 in human oocytes were predicted. SMARCA4 affects molecules indicated at the end of arrows, blue arrows mean 'Leads to inhibition', black arrows mean 'Effect not predicted'. Molecules colored green, red and blue show 'downregulated', 'upregulated', and 'predicted inhibition', respectively. 

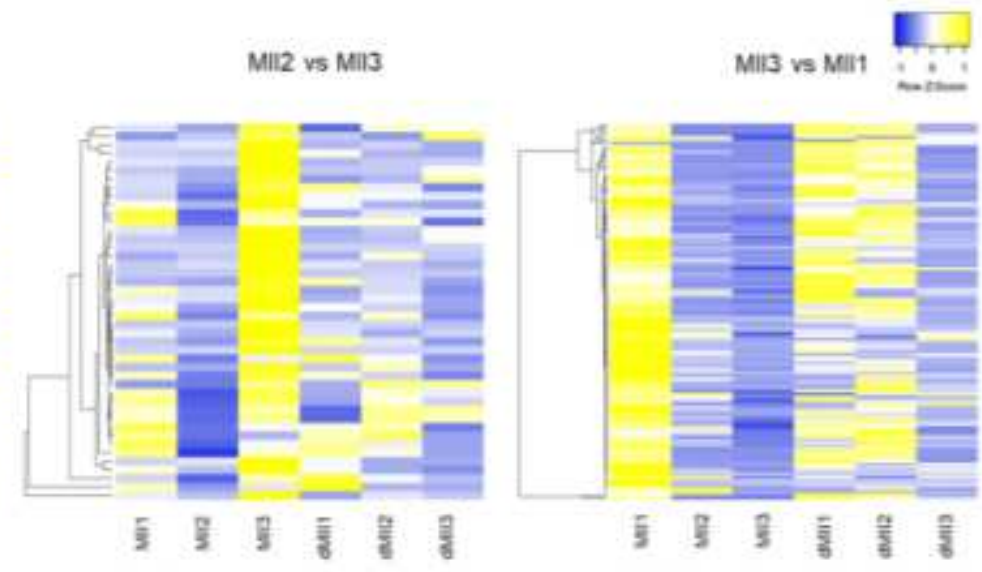

b Heatmaps of downregulated genes Mil1 vs MII2
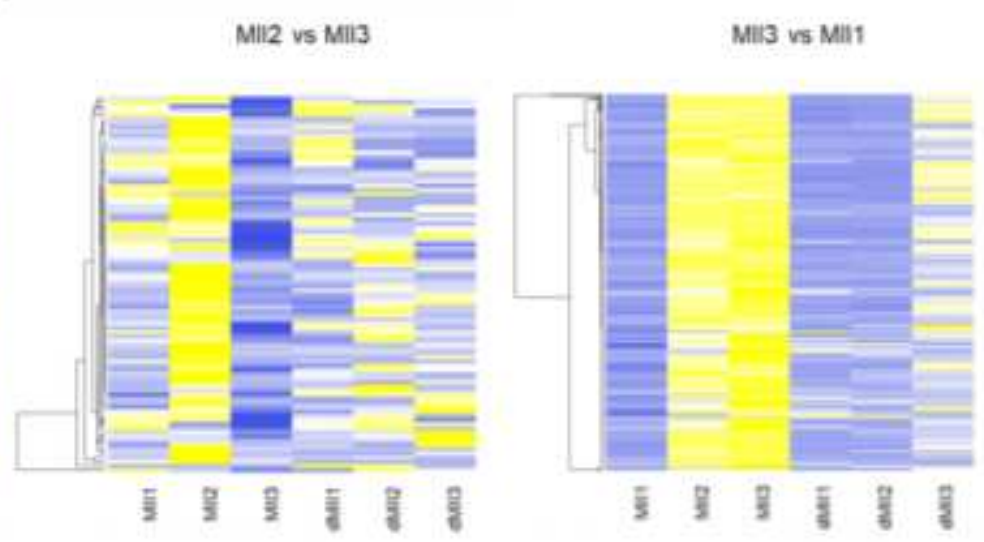

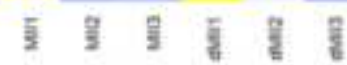
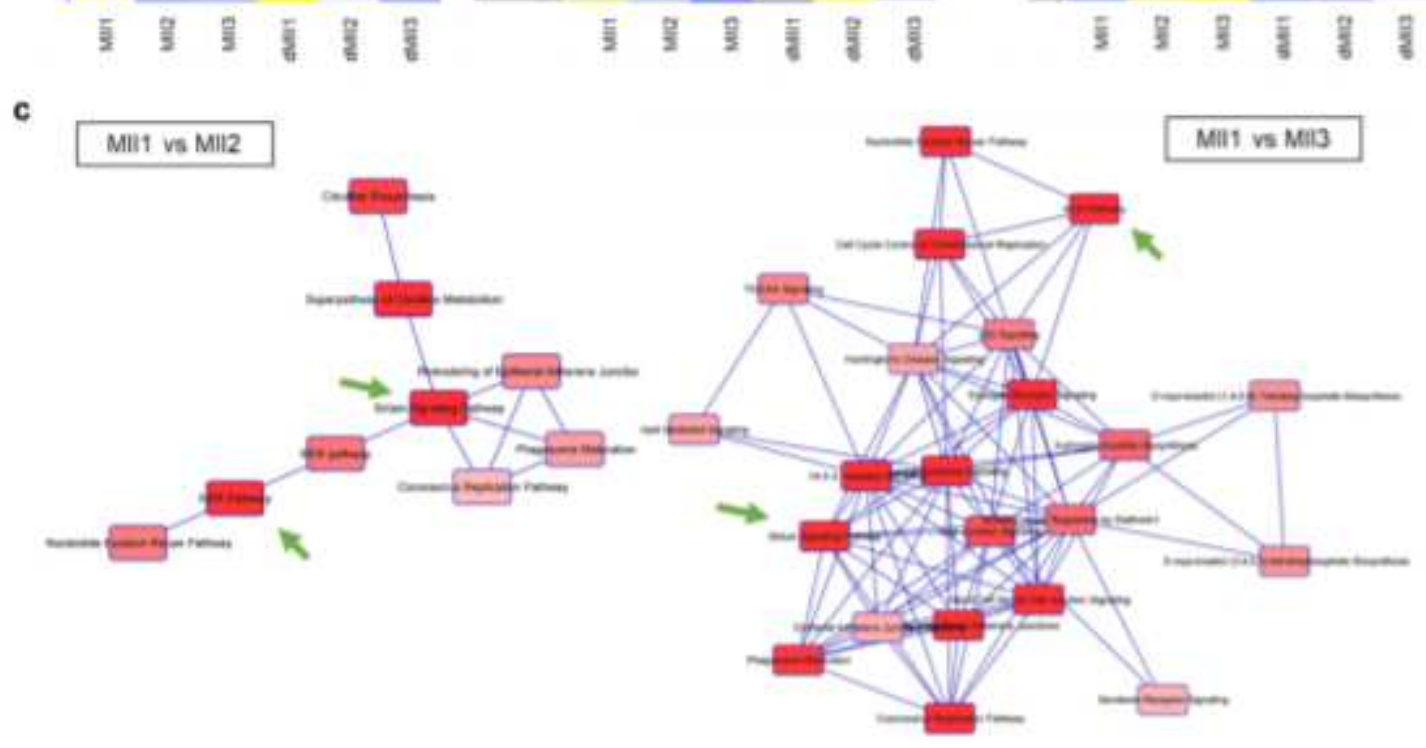

Figure 5

Gene regulatory networks that are altered in different patients. a, b Heatmaps depicting the gene expression levels of DEGs identified in the indicated comparisons. (a) shows upregulated genes in MII2 (MII1 vs MII2), MII3 (MII2 vs MII3), and MII1 (MII3 vs MII1) samples, while (b) indicates downregulated genes in the same comparisons. Color key with z-score is shown. c Canonical pathways predicted by IPA using the DEG list of MII1 vs MII2 or MII1 vs MII3. Significant terms are indicated in red colors: darker the color more significant the term is ( $P<0.05$, Fisher's exact test). Closely related terms are connected to each other. Green arrows indicate commonly misregulated pathways. 

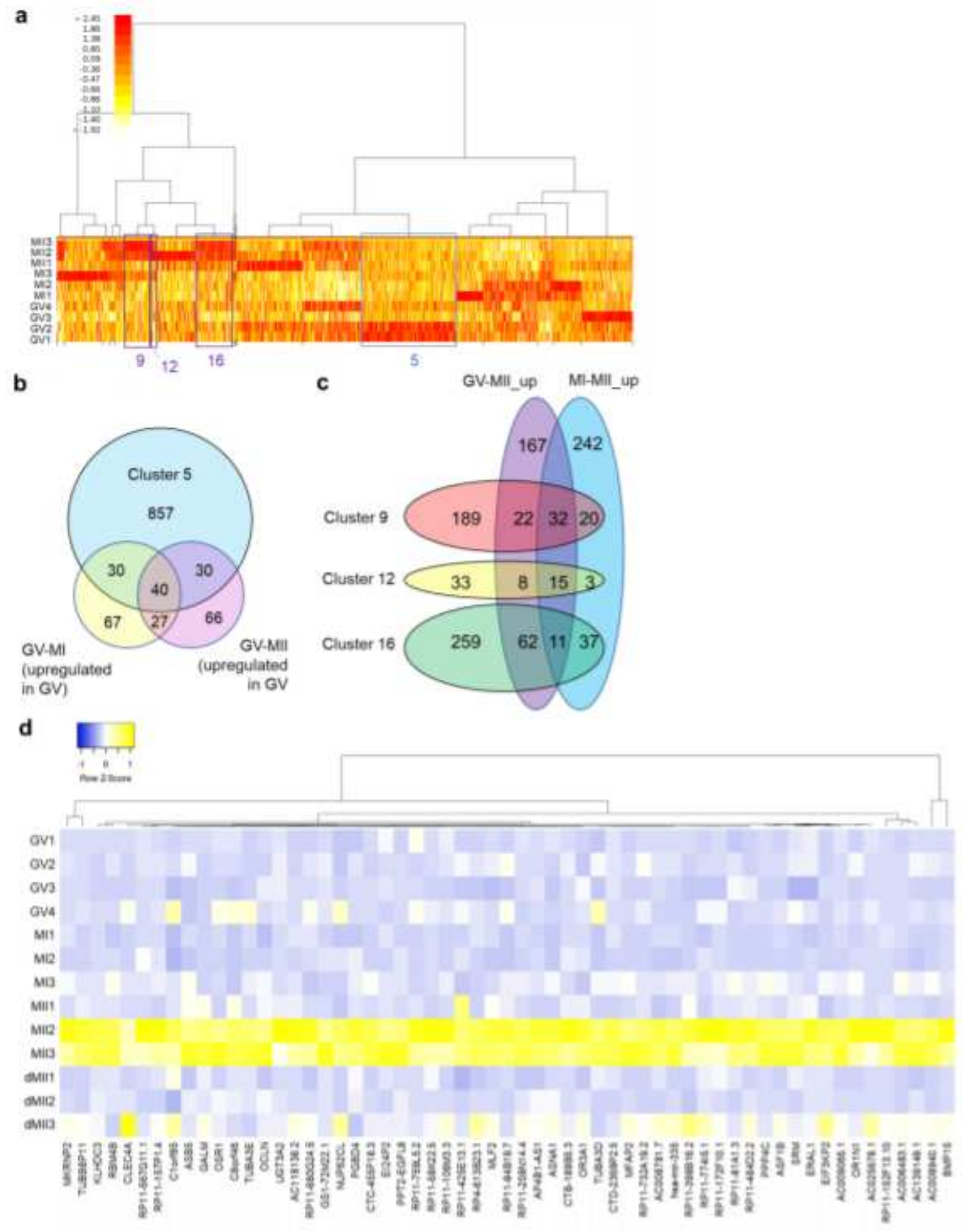

Figure 6

Marker genes for human GV and MII oocytes and potential biomarkers for MII oocyteswith high quality. a A heatmap of gene expression levels after clustering based on expression patterns. Twenty clusters were generated and clusters 5, 9, 12, and 16 are indicated. Color key indicates log2FC(FoldChange). b Venn diagrams showing the numbers of total and overlapping genes among genes that belong to cluster 5 , upregulated genes in the GV vs MI comparison, and upregulated genes in the GV vs MIl comparison. C Venn diagrams showing the numbers of total and overlapping genes among genes that belong to 
clusters 9,12 , and 16, upregulated genes in the GV vs MII comparison, and upregulated genes in the MI vs MII comparison. $\mathrm{d}$ A heatmap depicting the gene expression levels of candidate genes for human MII oocytes with high quality. Gene symbols are listed. Color key with z-score is shown.

\section{Supplementary Files}

This is a list of supplementary files associated with this preprint. Click to download.

- Supplementaryinformation.pdf

- TableS1.xlsx

- TableS2.xIsx

- TableS3.xIsx

- TableS4.xIsx

- TableS5.xIsx

- TableS6.xlsx

- TableS7.xlsx

- TableS8.xIsx

- TableS9.xIsx

- TableS10.xlsx 